Homology, Homotopy and Applications, vol.7(2), 2005, pp.5-39

\title{
CHICKEN OR EGG? A HIERARCHY OF HOMOTOPY ALGEBRAS
}

\author{
FÜSUN AKMAN
}

(communicated by James Stasheff)

\begin{abstract}
We start by clarifying and extending the multibraces notation, which economically describes substitutions of multilinear maps and tensor products of vectors. We give definitions and examples of weakly homotopy algebras, homotopy Gerstenhaber and Gerstenhaber bracket algebras, and homotopy Batalin-Vilkovisky algebras. We show that a homotopy algebra structure on a vector space can be lifted to its Hochschild complex, and also suggest an induction method to generate some of the explicit (weakly) homotopy Gerstenhaber algebra maps on a topological vertex operator algebra (TVOA), their existence having been indicated by Kimura, Voronov, and Zuckerman in 1996 (later amended by Voronov). The contention that this is the fundamental structure on a TVOA is substantiated by providing an annotated dictionary of weakly homotopy BV algebra maps and identities found by Lian and Zuckerman in 1993.
\end{abstract}

\section{Introduction}

The present paper originated from the author's confusion about the proliferation of G-objects, especially on a TVOA, and a desire to develop and clarify the multibraces language $([\mathbf{2}],[\mathbf{3}])$ to the point that it effortlessly describes and advances most homotopy algebra structures so far discovered. Pairs of braces \{\}\{\} , as well

as \{\}$\{, \ldots$,$\} were introduced by Gerstenhaber [6], and much later revived in$ more general form by Kadeishvili and Getzler. Our multibraces are a generalization of these braces, allowing one to substitute any number of maps into each other. Moreover, by defining the symbol $\left\{a_{1}, \ldots, a_{n}\right\}$ to be the tensor product of the vectors $a_{1}, \ldots, a_{n}$ (as it should be), we extend the idea of substitutions of maps from the tensor space $T A$ to the vector space $A$, and of vectors into these maps, to substitutions and tensor products in $\operatorname{Hom}(T A, T A)$. Whenever multilinear maps are defined on $\operatorname{Hom}(T A, A)$ (or $\operatorname{Hom}(T A, T A)$ ) itself, we use the "second-level braces" $[2]$ with primes (see Subsection 2.4). Section 2 gives an arguably self-contained and slightly improved account of the multibraces notation. In particular, substitution

Received December 2, 2003, revised September 30, 2004; published on April 22, 2005.

2000 Mathematics Subject Classification: 18G50, 18G55.

Key words and phrases: Batalin-Vilkovisky algebras, homotopy algebras.

(C) 2005, Füsun Akman. Permission to copy for private use granted. 
operators are now treated from the viewpoint of order-preserving maps between finite sets and of contractions of mixed tensors as well. A new insight into partitioned maps is provided via finite ordered partitioned (FOP) sets and cell-order preserving (COP) maps between them.

Following the work of Kimura, Voronov, and Zuckerman [13] in 1996, we were able to reduce the defining identities of a certain weakly homotopy Gerstenhaber algebra structure on a topological vertex operator algebra (TVOA) to a statement about the vanishing of a composition of mega operators. Later, Voronov [28] made some corrections to $[\mathbf{1 3}]$, and as a result, our construction was only partially applicable to the TVOA (although the same mega identity continues to fit perfectly the structure on the Hochschild complex of an associative algebra).

We will continue to regard the construction in $[\mathbf{1 3}]$ and $[\mathbf{2 8}]$ as a weakly homotopy Gerstenhaber algebra. Unless specifically designated "strongly" homotopy algebras (an operadic, or according to M. Markl, "God-given", property), all homotopy algebras mentioned in the article are "weakly" homotopy algebras. That is, they have the algebraic property that some of the constituent maps descend to a classical structure in the cohomology. Moreover, "Gerstenhaber" and "Batalin-Vilkovisky" will be shortened to $\mathrm{G}$ and BV respectively, where appropriate.

We are finally in a position to tie the intriguing weakly homotopy BatalinVilkovisky algebra structure on a TVOA, discovered several years earlier by Lian and Zuckerman [19] and published in 1993, to weakly homotopy Gerstenhaber algebras in an explicit manner. True to the chronological order, it has been conjectured in [13] that the anti-symmetrization of the homotopy BV maps of Lian and Zuckerman (chicken) will lay some fresh homotopy Gerstenhaber maps (eggs). We refer the reader to Huang and Zhao's article [10] on a new operadic and geometric formulation of a TVOA and the proof of the conjecture by Lian and Zuckerman and Kimura et al. stating that a TVOA gives rise to a homotopy Gerstenhaber algebra. It turns out that the egg is the parent of the chicken after all; we can now identify some components of the lower identities in [19] as bona fide weakly homotopy G-operators in the sense of $[\mathbf{1 3}]$ and $[\mathbf{2 8}]$. In fact, every explicit map introduced by Lian and Zuckerman as part of the weakly homotopy BV structure is simply the bracket of the anti-ghost operator $b_{0}$ with the homotopy G-maps, and every explicit identity is either homotopy Gerstenhaber, or can be obtained from one by bracketing with $b_{0}$ : the correspondence becomes visible in [19] once each identity is deciphered as "sums of compositions of maps equals zero". (Lian and Zuckerman's work also gives us an idea as to how to construct some of the homotopy G-algebra maps on a TVOA explicitly by induction.) Subsections 4.1.2, 4.2.2, and 4.2.3, as well as Appendix B, are devoted to the properties of TVOA's.

Then why not define a weakly homotopy BV algebra to be in general an algebra obtained by bracketing homotopy G-maps with a suitable odd operator? (See Tamarkin and Tsygan's definitive work on $B V_{\infty}$ algebras [26].) Apart from establishing reasonable definitions of several types of weakly homotopy algebras (Subsections $3.2,3.3$, and 3.5 , Section 5 ), we show that any generic homotopy algebra structure on a space $A$ can be lifted to its Hochschild complex, $C^{\bullet}(A, A)=\operatorname{Hom}(T A, A)$, in Subsection 4.2.1 (Getzler has a similar construction for $A_{\infty}$ algebras in [8]). The 
original homotopy G-algebra structure on the Hochschild complex is discussed in Subsection 4.1.1, and the novel role of new symmetry operators (such as the one that generates commutativity) in constructing self-contained mega identities is explored in Subsection 2.3.

Throughout the paper we point to the dichotomy between mega maps that are called "(weakly) homotopy" because the structure descends to the classical case in the cohomology, and mega maps that should be called "homotopy" because they satisfy identities similar to the classical case (and descend to it in the cohomology as well). A familiar example of coexistence of these phenomena is that we regard an $A_{\infty}$ algebra to be a homotopy associative algebra because the map $m_{(2)}$ descends to an ordinary associative algebra map in the $m_{(1)}$-cohomology, but also because its mega map $m$ satisfies the same identity as $m_{(2)}$, namely that $m_{(2)}$ composed with itself is zero. Thus certain homotopy Gerstenhaber algebras are given that name through the anti-symmetrization of their special bilinear maps $m_{(1 \mid 1)}$ descending to a G-bracket; yet others could be called G-algebras because their mega maps would be composed of higher brackets satisfying certain familiar identities, one of which directly descends to a G-bracket. We will see that a TVOA becomes a homotopy G-algebra in two different ways. Our notation $m_{(1 \mid 1)}$ will mostly refer to the composition operator between two multilinear maps and occasionally to abstract bilinear operators with similar properties.

Our goals are, then, (a) to improve and summarize the multibraces notation of the author, in order to simplify language and engender new identities and proofs, (b) to generate modified definitions of weakly homotopy algebras, involving maps that correspond to symmetries, as well as mega maps that behave like their classical counterparts, (c) to extend any generic homotopy algebra structure under the new definition to the Hochschild complex of the underlying vector space, (d) to dissect the homotopy BV maps in Lian-Zuckerman's paper and show that they should antecede the homotopy G maps, and (e) to hatch discussions of terminology and inspirations for new examples of homotopy algebras.

We will not make many references to operads in the present article; the exposition by Jones in $[\mathbf{1 1}]$ is recommended for a very refined treatment of substitution operators, and much more, in the context of operads. All our algebraic structures will be defined on a real or complex vector space with one or more gradings to be discussed later.

\section{Composition operators and relations}

\subsection{Standard composition: 3 viewpoints}

\subsubsection{Original definition}

The following is intended as a brief reminder of how to compose several multilinear maps with one (using the notation of braces), an idea that goes back to Gerstenhaber's seminal paper [6]. The expression [2]

$$
\{x\}\left\{y_{1}, \ldots, y_{k}\right\} \cdots\left\{z_{1}, \ldots z_{l}\right\}\left\{a_{1}, \ldots, a_{n}\right\}
$$

where the $a_{i}$ 's are vectors in a vector space $A$ and the remaining letters denote specific multilinear maps, possibly vectors, means that every symbol except $x$ is to 
be substituted into one on the left, in every possible way, but two vectors as well as two symbols within the same pair of parentheses may not be substituted into one another during this process. The resulting substitutions may not change the order within any one pair of braces, either. The signed sum of all such expressions are then formed, taking into consideration the bidegrees of the symbols that have been interchanged with respect to the original above.

We define the plus/minus sign picked up in the exchange of maps (possibly vectors) $x$ and $y$ to be

$$
(-1)^{|x||y|+d(x) d(y)},
$$

where $|x|$ denotes the super degree of the object $x$ (if any) and $d(x)$ denotes the number of arguments of $x$ minus one. In particular, $d(x)=0$ for a linear map $x$ and $d(x)=-1$ if $x$ is a vector. Both degrees are preserved in compositions of all types.

Note that although we require there be enough combined arguments to accommodate all symbols to the right, we do not require an exact match. In other words, there may be some free arguments (to the left of the string of $a_{i}$ 's) that would give us the flexibility to slide the multilinear maps within the boundaries of the pecking order. Also note that the $a_{i}$ 's are not an essential part of braces, just as the composition $f \circ g$ of ordinary functions is meaningful on its own without an argument. If included, the $a_{i}$ 's are frequently used to display the number (and grouping) of arguments of the composite multilinear functions to their left. Let us establish here the convention of reserving the symbols $a_{i}$ (or $a, b, c, \ldots$ ) for this exclusive purpose.

We also define the Gerstenhaber bracket ( $G$-bracket) of two maps $x$ and $y$ by

$$
[x, y]=\{x\}\{y\}-(-1)^{|x||y|+d(x) d(y)}\{y\}\{x\},
$$

as Gerstenhaber did in [6]. If, say, $x$ is a vector, then $\{x\}\{y\}$ will be zero. It is possible to remove this condition and allow all compositions between mixed tensors (with any number of covariant and contravariant factors).

Example 2.1. For a trilinear map $x$ and linear and four-linear maps $y, z$ respectively, the substitution $\{x\}\{y, z\}$ is given by

$$
\begin{aligned}
& \{x\}\{y, z\}\left\{a_{1}, a_{2}, a_{3}, a_{4}, a_{5}, a_{6}\right\} \\
= & -(-1)^{\left|a_{1}\right||z|} x\left(y\left(a_{1}\right), z\left(a_{2}, a_{3}, a_{4}, a_{5}\right), a_{6}\right) \\
+ & (-1)^{\left(\left|a_{1}\right|+\left|a_{2}\right|\right)|z|} x\left(y\left(a_{1}\right), a_{2}, z\left(a_{3}, a_{4}, a_{5}, a_{6}\right)\right) \\
+ & (-1)^{\left|a_{1}\right|(|y|+|z|)+\left|a_{2}\right||z|} x\left(a_{1}, y\left(a_{2}\right), z\left(a_{3}, a_{4}, a_{5}, a_{6}\right)\right) .
\end{aligned}
$$

Example 2.2. $\{x\}\{y\}\{z\}$ means that $y$ is substituted into $x$ in all possible ways and $z$ may either go into $y$ or straight into $x$. That is, we have

$$
\{x\}\{y\}\{z\}=\{x\}\{\{y\}\{z\}\}+\{x\}\{y, z\} \pm\{x\}\{z, y\} .
$$

Example 2.3. Let $m$ be an even bilinear map. The identity $\{m\}\{m\}=0$ is nothing but the associativity of $m$ :

$$
\{m\}\{m\}\left\{a_{1}, a_{2}, a_{3}\right\}=m\left(m\left(a_{1}, a_{2}\right), a_{3}\right)-m\left(a_{1}, m\left(a_{2}, a_{3}\right)\right)=0 .
$$


Example 2.4. By definition, $\{m\}\left\{a_{1}\right\}\left\{a_{2}\right\}\left\{a_{3}\right\}$ denotes the anti-symmetrization of the expression $\{m\}\left\{a_{1}, a_{2}, a_{3}\right\}$ for a trilinear map $m$ :

$$
\{m\}\left\{a_{1}\right\}\left\{a_{2}\right\}\left\{a_{3}\right\}=\sum_{\sigma \in S_{3}} \operatorname{sgn}(\sigma) m\left(a_{\sigma(1)}, a_{\sigma(2)}, a_{\sigma(3)}\right),
$$

where $S_{3}$ denotes the symmetric group on three letters. If there is a super degree, a factor of $(-1)^{\left|a_{i}\right|\left|a_{j}\right|}$ is to be inserted for every crossing of symbols $a_{i}, a_{j}$.

The single composition \{\}\{\} gives a right pre-Lie product on the Hochschild space. Gerstenhaber proved this result in detail in [6], but see $[\mathbf{2}]$ for a very brief proof based on the new braces notation. As a result, the Gerstenhaber bracket makes the Hochschild complex into a bigraded Lie algebra, with respect to the super grading mentioned above (if any) and the $d$-grading. (A bilinear product $\star$ on a super graded vector space is called right pre-Lie if

$$
(a \star b) \star c-a \star(b \star c)=(-1)^{|b||c|}((a \star c) \star b-a \star(c \star b))
$$

holds for all homogeneous $a, b, c$.)

We finally note that there is an associative, commutative product defined by

$$
(n) *(k)=(n+k-1)
$$

on multilinear function types: the symbol $(n)$ refers to an $n$-linear map and the product gives us the type of the composite map. The notation $x(n)$ will denote an n-linear map $x$ from now on, hence the composition of $x(n)$ and $y(k)$ will result in some $z(n+k-1)$ in accordance with the above rule. We will later extend this multiplication to ordered partitions, also to be considered as function types.

2.1.2. $\quad$ Order preserving maps between finite ordered sets

The notion that substitutions of many multilinear maps into one correspond to order-preserving functions between finite sets is not a new one (see [11] and [14]).

Example 2.5. The function

$$
f:\{1,2,3,4,5,6\} \rightarrow\{1,2,3,4,5\}
$$

given by

$$
f(1)=f(2)=1, f(3)=4, f(4)=f(5)=f(6)=5
$$

is an order-preserving map that has the inverse images

$$
\{1,2\}, \emptyset, \emptyset,\{3\},\{4,5,6\}
$$

for the five elements in the range respectively. Then $f$, as the unique disjoint union of five constant maps (some of which are missing a domain), represents the substitution of a bilinear map into the first argument of a five-linear map, together with the substitution of a linear map into the fourth argument and of a trilinear map into the fifth argument.

We hereby devise the following notation to denote substitutions without resorting to balloon diagrams and arrows: 
Example 2.6. The arrangement

$$
((2), 2,(1),(3)) \quad(\text { or }((2), 1,1,(1),(3)))
$$

shows a certain rigid placement of multilinear maps (say) $y(2), z(1)$, and $w(3)$ into some $x(5)$, the loose integer 2 denoting the two arguments of $x(5)$ that remain unoccupied. The resulting composite map is 8-linear, or of type (8), as can be seen by removing inner parentheses and replacing commas by plus signs.

Example 2.7. The symbol ((2), (1),4,(3),(0) ) denotes the substitution of a bilinear map and a linear map into the first two arguments of an 8-linear map, followed by four blank spaces, a trilinear map, and a specific vector. The final map has room for $2+1+4+3+0=10$ vectors.

Braced compositions are nothing but sums of the rigid substitutions described here, where the leftmost map is represented by outer parentheses and spaces between commas in the new notation, and more than two nested substitutions are left out for simplicity (but the notation makes it very clear as to how to proceed in case of additional nesting). Let us call each of these rigid substitutions a composition operator.

Example 2.8. If we wanted to describe the entire composition

$$
\{x(5)\}\{y(2), z(1), w(3)\},
$$

then we would have to write the sum

$$
\begin{aligned}
& ((2),(1),(3), 2)+((2),(1), 2,(3))+((2), 2,(1),(3))+(2,(2),(1),(3)) \\
+ & ((2),(1), 1,(3), 1)+((2), 1,(1),(3), 1)+(1,(2),(1),(3), 1) \\
+ & ((2), 1,(1), 1,(3))+(1,(2),(1), 1,(3))+(1,(2), 1,(1),(3))
\end{aligned}
$$

of specific composition operators.

The meta-composition of two composition operators (equivalently, of two orderpreserving functions) gives us another composition operator, and has a simple rule. First consider two operators $S=\left(\left(n_{1}\right), \ldots,\left(n_{k}\right)\right)$ and $T=\left(\left(m_{1}\right), \ldots,\left(m_{l}\right)\right)$, where all entries are within parentheses, and $n_{1}+\cdots+n_{k}=l$. Their composition is given by the new operator

$$
S \circ T=\left(\left(\sum_{i=1}^{n_{1}} m_{i}\right),\left(\sum_{i=1}^{n_{2}} m_{n_{1}+i}\right), \ldots,\left(\sum_{i=1}^{n_{k}} m_{n_{1}+\cdots+n_{k-1}+i}\right)\right),
$$

that is, we add up the first $n_{1}$ entries on the right, then the next $n_{2}$, and so on. When $n_{i}=0$ we have an empty sum and the resulting entry is $(0)$.

\section{Example 2.9.}

$$
((2),(1),(3),(0)) \circ((1),(0),(3),(0),(2),(4))=((1),(3),(6),(0)) .
$$

In case the composition operator on the left involves positive integers $r$ without parentheses, these numbers are to be copied as is, since they correspond to unoccupied arguments. 
Example 2.10.

$$
((2),(1), 3) \circ((1),(5),(4))=((6),(4), 3) .
$$

Similar numbers $r \geqslant 1$ on the right should be converted to $r$ 1's separated by commas, and then the usual rules should be applied, as if all entries are surrounded by parentheses.

\section{Example 2.11.}

$$
((1),(3)) \circ((2),(4), 2)=((1),(3)) \circ((2),(4), 1,1)=((2),(6)) .
$$

We should note here that the convention of drawing arrows representing functions from left to right in diagrams is the opposite of the braces convention as well as the usual $f \circ g$ notation. In [14], for example, the notation for substitutions is changed from $f x$ to $x f$ to match arrow directions in the pictures.

In the subsections on partitioned maps, we will denote the meta-type of any substitution operator of $n$ maps into one by $(1 \mid n)$ (regardless of the types of the little argument-maps inside) and underline the composition of two such operators by a product $(1 \mid n) *(1 \mid k)$.

\subsubsection{Contractions}

Just as a linear map $f: A \rightarrow A$ is an element of $A^{*} \otimes A$, a substitution $f(a)$ is the contraction of the previous tensor with $a$. Let us expand this idea to all substitutions. For demonstration purposes -all our definitions will be basis free- fix a basis $\left\{a_{i}\right\}$ for a finite dimensional vector space $A$ and let $\left\{a_{i}^{*}\right\}$ denote the dual basis for $A^{*}$ (this amounts to endowing $A$ with a Frobenius algebra structure; see [14]). The notion of composition, or multiple compositions, on the Hochschild space

$$
T_{\bullet}^{1} A=\bigoplus_{q \geqslant 0} T_{q}^{1} A=\bigoplus_{q \geqslant 0}\left(A^{*}\right)^{\otimes q} \otimes A=\bigoplus_{q \geqslant 0} \operatorname{Hom}\left(A^{\otimes q} ; A\right)
$$

is naturally extended to the space

$$
T_{\bullet}^{\bullet} A=\bigoplus_{p, q \geqslant 0} T_{q}^{p} A=\bigoplus_{p, q \geqslant 0}\left(A^{*}\right)^{\otimes q} \otimes A^{\otimes p}=\bigoplus_{p, q \geqslant 0} \operatorname{Hom}\left(A^{\otimes q} ; A^{\otimes p}\right)
$$

of tensors of all types on $A$ as described below. The notational convention is borrowed from $[\mathbf{2 7}]$.

The space of mixed tensors of type $(p, q)$, denoted by $T_{q}^{p} A$, is spanned by basis elements of the form

$$
a_{i_{1}}^{*} \otimes \cdots \otimes a_{i_{q}}^{*} \otimes a_{j_{1}} \otimes \cdots \otimes a_{j_{p}} .
$$

Let us denote the corresponding elements in the isomorphic space $\operatorname{Hom}\left(A^{\otimes q} ; A^{\otimes p}\right)$ by

$$
T_{i_{1} \ldots i_{q}}^{j_{1} \ldots j_{p}}
$$

where

$$
\begin{aligned}
T_{i_{1} \ldots i_{q}}^{j_{1} \ldots j_{p}}\left(a_{n_{1}} \otimes \cdots \otimes a_{n_{q}}\right) & =a_{i_{1}}^{*}\left(a_{n_{1}}\right) \cdots a_{i_{q}}^{*}\left(a_{n_{q}}\right) a_{j_{1}} \otimes \cdots \otimes a_{j_{p}} \\
& =\delta_{i_{1} n_{1}} \cdots \delta_{i_{q} n_{q}} a_{j_{1}} \otimes \cdots \otimes a_{j_{p}} .
\end{aligned}
$$


The latter (map) form is useful in depicting multilinear maps with values in $A$, whereas the former (tensor) notation will provide the best framework to extend substitution to the space $T \bullet A$. In fact, given the previous usage of multibraces, let us use the notation

$$
\left\{a_{i_{1}}^{*}, \ldots, a_{i_{q}}^{*} ; a_{j_{1}}, \ldots, a_{j_{p}}\right\}
$$

for the tensor version of the general basis element. If the contravariant or covariant factors are completely missing, then we will substitute the symbol $\emptyset$ for that part and still separate the two sets by a semicolon: for example, we will have

$$
a \otimes b=\{\emptyset ; a, b\} \in T_{0}^{2} A
$$

for $a, b \in A$. We will occasionally use general elements of the algebra (denoted by $a, b, c$, etc.) instead of the basis elements and general functionals $(f, g, h$, etc.) instead of the dual basis elements. For example, a linear map on $A$ is a finite sum of irreducible tensors $F=\{f ; a\}$ where

$$
F(b)=f(b) a .
$$

A bilinear map with values in $A$ is similarly a finite sum of tensors $G=\{f, g ; a\}$ where

$$
G(b, c)=f(b) g(c) a .
$$

Example 2.12. In tensor notation, it suffices to show the substitution of one trilinear map $_{(3)}$ into a bilinear map $m_{(2)}$ for irreducible tensors

$$
m_{(2)}=\{f, g ; u\} \quad \text { and } \quad x_{(3)}=\{h, k, l ; v\} .
$$

We claim that

$$
\{f, g ; u\} \circ\{h, k, l ; v\}=f(v)\{h, k, l, g ; u\} \pm g(v)\{f, h, k, l ; u\},
$$

with

$$
\begin{aligned}
& (\{f, g ; u\} \circ\{h, k, l ; v\})(a, b, c, d) \\
= & (f(v)\{h, k, l, g ; u\} \circ\{\emptyset ; a, b, c, d\} \pm g(v)\{f, h, k, l ; u\} \circ\{\emptyset ; a, b, c, d\} \\
= & f(v) h(a) k(b) l(c) g(d)\{\emptyset ; u\} \pm g(v) f(a) h(b) k(c) l(d)\{\emptyset ; u\} .
\end{aligned}
$$

Note that the first (covariant) part of the first tensor is contracted with the second (contravariant) part of the second tensor. In fact, the composition operator will turn out to be nothing but contraction among all types of tensors. The equivalence of the above expression to the original definition can be seen from

$$
\begin{aligned}
& m_{(2)}\left(x_{(3)}(a, b, c), d\right) \pm m_{(2)}\left(a, x_{(3)}(b, c, d)\right) \\
= & m_{(2)}(h(a) k(b) l(c) v, d) \pm m_{(2)}(a, h(b) k(c) l(d) v) \\
= & h(a) k(b) l(c) m(2)(v, d) \pm h(b) k(c) l(d) m(2)(a, v) \\
= & h(a) k(b) l(c) f(v) g(d) u \pm h(b) k(c) l(d) f(a) g(v) .
\end{aligned}
$$

The contraction rule we have used in the composition operator $\circ=M_{(1 \mid 1)}$, defined on the Hochschild space of multilinear maps on $A$ with values in $A$, is as follows 
(note the usage of a bar that separates the composed multilinear maps, mimicking the bar in "type" $(1 \mid 1))$ :

$$
\begin{aligned}
& M_{(1 \mid 1)}\left(\left\{f_{1}, \ldots, f_{m} ; b\right\} \mid\left\{g_{1}, \ldots, g_{n} ; c\right\}\right) \\
= & \sum_{i=1}^{m} \pm f_{i}(c)\left\{f_{1}, \ldots, f_{i-1}, g_{1}, \ldots, g_{n}, f_{i+1}, \ldots, f_{m} ; b\right\} .
\end{aligned}
$$

The rule, generalized to all tensor types, can be grouped into four types for convenience:

$$
M_{(1 \mid 1)}\left(\left\{f_{1}, \ldots, f_{m} ; b_{1}, \ldots, b_{k}\right\} \mid\left\{g_{1}, \ldots, g_{n} ; c_{1}, \ldots, c_{l}\right\}\right)
$$

is given by

$$
f_{1}\left(c_{1}\right) \cdots f_{m}\left(c_{m}\right)\left\{g_{1}, \ldots, g_{n} ; b_{1}, \ldots, b_{k}\right\}
$$

if $m=l$ (the strings of $f$ 's and c's have the same nonzero length);

$$
\sum_{i=1}^{m-l+1} f_{i}\left(c_{1}\right) \cdots f_{i+l-1}\left(c_{l}\right)\left\{f_{1}, \ldots, f_{i-1}, g_{1}, \ldots, g_{n}, f_{i+l}, \ldots, f_{m} ; b_{1}, \ldots, b_{k}\right\}
$$

if $m>l \geqslant 1$ (there are more $f$ 's than $c^{\prime}$ s); and

$$
\sum_{i=1}^{l-m+1} f_{1}\left(c_{i}\right) \cdots f_{m}\left(c_{i+m-1}\right)\left\{g_{1}, \ldots, g_{n} ; c_{1}, \ldots, c_{i-1}, b_{1}, \ldots, b_{k}, c_{i+m} \ldots, c_{l}\right\}
$$

if $l>m \geqslant 1$ (there are more c's than $f$ 's). If either $m=0$ or $l=0$, then the result is understood to be zero. That is, purely contravariant tensors on the left do not accept substitutions and purely covariant tensors on the right cannot be substituted into anything. These rules are in perfect harmony with our previous definitions.

In short, the contraction is achieved by sliding the shorter of the strings $f, c$ along the longer one and adding up. The empty sum is zero. When writing the nonconstant portion of the result, the part of the longer string (e.g. $f$ 's) that is used in the contraction is replaced by its counterpart (e.g. $g$ 's) in the other tensor. Order is important!

Example 2.13. Using the third one of these four rules, it is easy to check that, for example,

$$
\left\{m_{(2)}\right\}\{a, b, c\}
$$

is given by

$$
m_{(2)}(a, b) \otimes c \pm a \otimes m_{(2)}(b, c)=f(a) g(b) u \otimes c \pm a \otimes f(b) g(c) u .
$$

Substitution of more arguments than what the left-hand map can take (as a block) is now allowed. Indeed, if we take $m_{(2)}$ to be $\{f, g ; u\}$, then we have

$$
M_{(1 \mid 1)}(\{f, g ; u\} \mid\{\emptyset ; a, b, c\})=f(a) g(b)\{\emptyset ; u, c\} \pm f(b) g(c)\{\emptyset ; a, u\}
$$

according to the generalized definition of substitution.

The preservation of the $d$-grading is even more obvious in contraction form. For an element of $T_{q}^{p} A, d$ is equal to $q-p$, or the "degree of covariance" of the tensor. 
With each contraction, equal numbers of covariant and contravariant factors are lost, which does not affect the sum of the $d$-gradings.

\subsection{Partitioned composition}

\subsubsection{Original definition}

The algebra of partitions and substitutions by partitioned braces first came up in the author's work describing a master identity for homotopy Gerstenhaber algebras [3] (later shown to be inadequate except for lower identities as a result of Voronov's amendment $[\mathbf{2 8}]$ to $[\mathbf{1 3}])$. They were the algebraic equivalent of the construction of the boundary (differential) in the pictures of Fox-Neuwirth cells in [13] and [28]. We will not demonstrate the brute-force way of composing "partitioned maps" $M_{\pi}$ of type $\pi=\left(n_{1}\left|n_{2}\right| \cdots \mid n_{k}\right)\left(n_{i}>0\right)$ in the text (it would be painful), but we do give some examples in Appendix A. We note the use of capital $M$ and the resemblance to the composition operators of type (1|1), but do not assume yet that these partitioned maps are really composition operators with arguments in the Hochschild complex or its extension.

A partitioned map $M_{\pi}$, where $\pi$ is the generic partition described above, is first and foremost a multilinear map on some vector space $A$ with $n=\sum n_{i}$ arguments. By itself, we do not attach any deeper meaning to the partition that is the type of $M$. But how do we compose a partitioned map (on the left) with another (on the right), or several others? Unfortunately, unlike compositions of maps of plain types, the multilinear maps that denote the results of all possible rigid substitutions will possibly have more than one type, and will have to be grouped according to these types (partitions). That is, if some $M_{\pi}$ is composed with some $N_{\tau}$, then we will end up with a sum of maps $X_{\sigma}$ of varying partition types $\sigma$, and necessarily subject to different substitution rules when composed with their ilk. Soon we will define precisely a product $\pi * \tau=\sigma+\cdots$ on partitions that extends the product $(n) *(k)=(n+k-1)$ and underlies the composition rule (see next subsection). We want to emphasize that this product, which governs types of maps resulting from partitioned compositions, is pre-Lie $([\mathbf{3}])$.

Partitions with 0's will be used to write the symmetries of a map. For example, a multilinear map of type $(1 \mid 0)$ is in fact a linear map that only acts on the first slot and ignores the slot immediately to the right. Since this notion deserves its own space, we will revisit it in Subsection 2.3.

\subsubsection{Cell-order preserving (COP) maps between finite ordered partitioned (FOP)} sets

We have replaced the notation $(n)$ denoting the type, or number of arguments, of a multilinear map by a partition $\pi=\left(j_{1}\left|j_{2}\right| \cdots \mid j_{s}\right)(s \geqslant 1)$, where the "parts", or "slots", $j_{1}, j_{2}, \ldots, j_{s}$ add up to $n$. This change, as we have noted, affects only the composition or substitution properties of the function.

We define, as we did in [3], the product between a single-slot partition and a general partition as follows:

$$
(k) *\left(n_{1}|\cdots| n_{j}\right)=\sum_{t_{1}, \ldots, t_{j} \geqslant 0, \sum t_{i}=k-1}\left(n_{1}+t_{1}|\cdots| n_{j}+t_{j}\right) .
$$


That is, we distribute the number $k-1$ among all slots and add the parts to the slots, in all possible ways.

Example 2.14.

$$
\begin{aligned}
(4) *(1 \mid 2) & =(1+0 \mid 2+3)+(1+1 \mid 2+2)+(1+2 \mid 2+1)+(1+3 \mid 2+0) \\
& =(1 \mid 5)+(2 \mid 4)+(3 \mid 3)+(4 \mid 2) .
\end{aligned}
$$

If the partition on the left has several slots, then we multiply each slot separately by the partition on the right, as described above, while leaving the remaining slots as they are and expanding linearly. Thus,

$$
\left(k_{1}|\cdots| k_{l}\right) * \pi \approx \sum_{t=1}^{l}\left(k_{1}|\cdots|\left(k_{t}\right) * \pi|\cdots| k_{l}\right),
$$

where the sign $\approx$ as opposed to $=$ means that any repetitions of the same term have to be ignored.

Example 2.15. We have

$$
(2 \mid 4) *(1 \mid 2) \approx(2|2| 4)+(1|3| 4)+(2|4| 2)+(2|3| 3)+(2|2| 4)+(2|1| 5),
$$

so the actual value of the product is

$$
(2 \mid 4) *(1 \mid 2)=(2|2| 4)+(1|3| 4)+(2|4| 2)+(2|3| 3)+(2|1| 5) .
$$

This means we may choose to treat the composition of a (2|4)-linear map with a (1|2)-linear map as (2|2|4)-linear, or (1|3|4)-linear, etc., and substitute suitably partitioned elements. In Appendix $A$ we show how to expand the substitution as a (2|2|4)-linear map.

We will now construct a new and simpler device to study the partitioned composition operators introduced in $[\mathbf{3}]$, modeled after the category of finite ordered sets and order-preserving maps. Let us take a unique representative of every finite, ordered, partitioned (FOP) set, such as $\{1|2,3,4| 5,6\}$. These sets can be arranged vertically, ordered from bottom to top, say, and certain functions between them easily visualized. We will call these functions cell-order preserving (COP), where a cell of a FOP set, similar to a "slot" or "part" in a partition, will simply mean the ordered set of elements between two bars. COP functions will have the following properties by definition, which can easily be checked to hold under compositions:

1. The function will preserve the order within each cell, but not necessarily within the entire domain.

2. The image of any cell in the domain will lie within only one cell in the range.

3. The pre-image of any full subset of a string of vertically adjacent cells in the range will either be the empty set or again a full subset of a string of adjacent cells in the domain.

(Here full subset is a term we have made up for the limited scope of describing a nonempty subset of a FOP set that lies within a string of adjacent cells and has nonempty, connected intersection with each of these cells. For example, $\{2,3 \mid 6\}$ is a full subset of $\{1,2,3,4 \mid 5,6,7\}$ but not of $\{1,2,3|4,5| 6,7\}$. A partial ordering can be 
imposed on the set of all full subsets of a FOP set. The phrase subset I is higher than subset II means all numerals in the first set are larger in value than the numerals in the second one.)

4. Arrows going to different cells in the range may not cross. That is, if cell I is higher than cell II in the range, then their pre-images will have the same ordering.

(For example, the function $f:\{1 \mid 2\} \rightarrow\{1 \mid 2\}$ defined by $f(1)=2$ and $f(2)=1$ is not COP.)

Additional properties that follow from the above are:

5. The pre-image of any singleton will be either the empty set or a full subset of a string of adjacent cells.

6. The pre-image of any string of adjacent cells (possibly one) will be either the empty set or a string of adjacent cells.

Disjoint unions of COP functions can be taken, as in the plain case, by vertically concatenating domains and ranges. It is clear that any COP function is in a unique way the disjoint union of a number of $\mathrm{COP}$ functions obtained by restricting the original function to the pre-image of each cell in the range. In particular, a COP function from one cell to another is a plain order-preserving (POP?) function. COP functions are then in one to one correspondence with all possible substitution types of several partitioned maps into one partitioned map: each cell in the range represents a plain multilinear map, whereas the pre-image of any singleton in that cell represents a partitioned multilinear map that is to be substituted into the argument marked by the singleton.

\subsection{Symmetries as funny composition operators}

Partitions with 0-slots may be used to obtain the symmetries among the generating identities of algebras in the following manner. It is our hope that this new approach will inspire fellow homotopicians to reconsider their favorite identities!

What is a symmetry? In our context, any generating identity that involves terms differing from each other only by permutations of the arguments (vectors) may be called a symmetry; in our experience, the permutations tend to occur within one map and not a composition of two or more maps (we know one when we see one!). For example, in a commutative associative algebra with bilinear map $m_{(2)}$, the commutativity identity $m_{(2)}(a, b)-m_{(2)}(b, a)=0$ is a symmetry, whereas the associativity identity $m_{(2)}\left(m_{(2)}(a, b), c\right)-m_{(2)}\left(a, m_{(2)}(b, c)\right)=0$ is not, since it exhibits no change of order in the arguments (also, there are two levels of maps in each term). We claim that this is only a superficial distinction. What if we define, in addition to $m_{(2)}$, a linear map $m_{(1 \mid 0)}$ that simply sends a vector to itself and is composed in the way indicated by the partition $(1 \mid 0)$ ? Then

$$
\left\{m_{(2)}\right\}\left\{m_{(1 \mid 0)}\right\}\{a \mid b\}
$$

is evaluated by placing $m_{(1 \mid 0)}$ into the first slot of $m_{(2)}$ only (the other possibility gives rise to the partitioning $(2 \mid 0)$ of the vectors, instead of (1|1)), and not choosing $b$ for substitution into the second slot of $m_{(1 \mid 0)}$ (some reading of Appendix A may be in order). We do not have many options as to how to substitute $a$ into the first slot, but in how many ways can we not substitute $b$ into the second? Let us split 
the $b$-slot into three sets in all possible manners:

$$
\{b\} \emptyset \emptyset \text {, or } \emptyset \emptyset\{b\} \text {. }
$$

Thus $b$ appears both as a left and a right leftover vector, and the evaluation is given by

$$
m_{(2)}\left(m_{(1 \mid 0)}(a \mid \emptyset), b\right)-m_{(2)}\left(b, m_{(1 \mid 0)}(a \mid \emptyset)\right)=m_{(2)}(a, b)-m_{(2)}(b, a)=0
$$

(it is much easier to visualize through diagrams of FOP sets and COP maps; left as an exercise!).

This manifestation of symmetry makes it possible for us to write all-quadratic expressions and use mega identities in a compact way. In particular, (weakly) homotopy Gerstenhaber algebras will be distinguished among all homotopy algebras by the inclusion of $m_{(1 \mid 0)}$ as the identity map described above. Thus we will be able to generalize the description of homotopy associativity of $A_{\infty}$ algebras to that of homotopy commutativity and associativity within the same identity.

\subsection{Isomorphism between Hochschild space and the space of coderiva- tions on the tensor coalgebra}

The use of multibraces, being very visual, simplifies the statements and proofs of many results. For future reference, and to see the effects of our notation in expanded settings, let us look into the isomorphism in the subsection heading. A primitive version of the following proof/explanation techniques first appeared in [2].

The second-level braces, namely those denoting tensor products and substitutions in the tensor algebra $T(T A)$, were also introduced in [2]. We distinguish this level by putting primes on the braces and the tensor product symbols. (In fact, we will use primed objects whenever multilinear maps are defined on the Hochschild complex itself.) Since we want to study coderivations and the comultiplication, it makes sense to consider maps from $T A=T^{1}(T A)$ into $T(T A)$. Let us now denote the tensor product $a_{1} \otimes \cdots \otimes a_{n}$ inside $T^{1}(T A)$ by

$$
\left\{\left\{a_{1}, \ldots, a_{n}\right\}\right\}^{\prime} .
$$

For example, the basis element

$$
\left(a_{1} \otimes \cdots \otimes a_{n}\right) \otimes^{\prime}\left(b_{1} \otimes \cdots \otimes b_{k}\right)
$$

of $T^{2}(T A)$ will be written as

$$
\left\{\left\{a_{1}, \ldots, a_{n}\right\},\left\{b_{1}, \ldots, b_{k}\right\}\right\}^{\prime} .
$$

Similarly, an old multilinear map $m$ will be denoted by $\{\{m\}\}^{\prime}$, and substitutions between symbols of the first level will still take place even if they are in different pairs of primed braces: we have

$$
\{\{m\}\}^{\prime}\left\{\left\{a_{1}, \ldots, a_{n}\right\}\right\}^{\prime}=\left\{\{m\}\left\{a_{1}, \ldots, a_{n}\right\}\right\}^{\prime}
$$

by definition. One such map we will use is the identity map

$$
i=i_{(0)}+i_{(1)}+i_{(2)}+\cdots+i_{(n)}+\cdots: T A \rightarrow T A
$$

where $i_{(n)}\left(a_{1} \otimes \cdots \otimes a_{n}\right)=a_{1} \otimes \cdots \otimes a_{n}$. "Primed" maps will be given capital letter names to distinguish them from the first-level maps. 
The standard associative bilinear product $M$ on $T A$,

$$
M: T^{1}(T A) \otimes T^{1}(T A) \rightarrow T^{1}(T A),
$$

is defined via $i$ :

$$
\begin{aligned}
& \{M\}^{\prime}\left\{\left\{a_{1}, \ldots, a_{n}\right\},\left\{b_{1}, \ldots, b_{k}\right\}\right\}^{\prime} \\
= & \{\{i\}\}^{\prime}\left\{\left\{a_{1}, \ldots, a_{n}\right\},\left\{b_{1}, \ldots, b_{k}\right\}\right\}^{\prime} \\
= & \left\{\left\{i_{(n+k)}\right\}\right\}^{\prime}\left\{\left\{a_{1}, \ldots, a_{n}\right\},\left\{b_{1}, \ldots, b_{k}\right\}\right\}^{\prime} \\
= & \left\{\left\{a_{1}, \ldots, a_{n}, b_{1}, \ldots, b_{k}\right\}\right\}^{\prime} .
\end{aligned}
$$

Meanwhile, the coassociative coproduct $\Delta$ on $T A$,

$$
\Delta: T^{1}(T A) \rightarrow T^{1}(T A) \otimes T^{1}(T A),
$$

has a similar definition:

$$
\begin{aligned}
& \{\Delta\}^{\prime}\left\{\left\{a_{1}, \ldots, a_{n}\right\}\right\}^{\prime} \\
= & \{\{i\},\{i\}\}^{\prime}\left\{\left\{a_{1}, \ldots, a_{n}\right\}\right\}^{\prime} \\
= & \sum_{u+v=n}\left\{\left\{i_{(u)}\right\},\left\{i_{(v)}\right\}\right\}^{\prime}\left\{\left\{a_{1}, \ldots, a_{n}\right\}\right\}^{\prime} \\
= & \sum_{u=0}^{n}\left\{\left\{a_{1}, \ldots, a_{u}\right\},\left\{a_{u+1}, \ldots, a_{n}\right\}\right\}^{\prime} .
\end{aligned}
$$

Then in accordance with the usual rules, we define derivations and coderivations of the bialgebra $T A$ as

$$
\operatorname{Der}(T A)=\left\{X \in \operatorname{Hom}\left(T^{1}(T A), T^{1}(T A)\right):[X, M]^{\prime}=0\right\}
$$

and

$$
\operatorname{Coder}(T A)=\left\{X \in \operatorname{Hom}\left(T^{1}(T A), T^{1}(T A)\right):[X, \Delta]^{\prime}=0\right\} .
$$

It is now easy to see why $C^{\bullet}(A, A)$ and $\operatorname{Coder}(T A)$ are naturally isomorphic. Given

$$
m=m_{(1)}+m_{(2)}+\cdots m_{(n)}+\cdots \in \operatorname{Hom}(T A, A),
$$

define $\delta(m) \in \operatorname{Coder}(T A)$ by

$$
\begin{aligned}
& \{\delta(m)\}^{\prime}\left\{\left\{a_{1}, \ldots, a_{n}\right\}\right\}^{\prime} \\
= & \{\{m\}\}^{\prime}\left\{\left\{a_{1}, \ldots, a_{n}\right\}\right\}^{\prime} \\
= & \sum_{k=1}^{n}\left\{\left\{m_{(k)}\right\}\right\}^{\prime}\left\{\left\{a_{1}, \ldots, a_{n}\right\}\right\}^{\prime} \\
= & \sum_{k=1}^{n} \sum_{j=1}^{n-k+1} \pm\left\{\left\{a_{1}, \ldots, m_{(k)}\left(a_{j}, \ldots, a_{j+k-1}\right), \ldots, a_{n}\right\}\right\}^{\prime} .
\end{aligned}
$$

Why is $\delta(m)$ a coderivation? We have

$$
\begin{aligned}
& \{\Delta\}^{\prime}\{\delta(m)\}^{\prime}\left\{\left\{a_{1}, \ldots, a_{n}\right\}\right\}^{\prime} \\
= & \{\{i\},\{i\}\}^{\prime}\{\{m\}\}^{\prime}\left\{\left\{a_{1}, \ldots, a_{n}\right\}\right\}^{\prime} .
\end{aligned}
$$


and

$$
\begin{aligned}
& \{\delta(m)\}^{\prime}\{\Delta\}^{\prime}\left\{\left\{a_{1}, \ldots, a_{n}\right\}\right\}^{\prime} \\
= & \{\{m\}\}^{\prime}\{\{i\},\{i\}\}^{\prime}\left\{\left\{a_{1}, \ldots, a_{n}\right\}\right\}^{\prime}
\end{aligned}
$$

In the first case, the first $n$ summands of the mega operator $m$ are applied in all possible ways to $\left\{a_{1}, \ldots, a_{n}\right\}$, then the resulting product is split into two in all possible ways as tensor products in $T^{2}(T A)$. In the second case, first $\left\{a_{1}, \ldots, a_{n}\right\}$ is split into two in all possible ways as tensor products in $T^{2}(T A)$, then the same summands of $m$ are applied in all possible ways to the split product. The end result is the same!

Going backwards from $\operatorname{Coder}(T A)$, we take any coderivation and compose it with the projection $T A \rightarrow A$, which gives a map in $\operatorname{Hom}(T A, A)$. Note that coderivations on $T A$ are uniquely determined by this projection, just as derivations would be uniquely determined by the restriction to $A$.

Note. The reason why Gerstenhaber's bracket on $\operatorname{Hom}(T A, A)$ is the same as the bracket of coderivations as linear maps is simply the following: both are written as $\{x\}\{y\} \pm\{y\}\{x\}$, and are subject to the multibraces rules.

\section{Homotopy algebras}

\subsection{Strongly homotopy associative, pre-Lie, and Lie algebras}

3.1.1. The suspended degree

Let $m$ be again a formal sum of infinitely many homogeneous maps in the Hochschild space $C^{\bullet}(A, A)$. We define another similar map, $\tilde{m}$, by changing each summand by a factor of \pm 1 . This factor will depend not only on the degree of homogeneity of the summand but also on the arguments of the map:

$$
\tilde{m}_{(k)}\left\{a_{1}, \ldots, a_{k}\right\}=(-1)^{(k-1)\left|a_{1}\right|+(k-2)\left|a_{2}\right|+\cdots+1 \cdot\left|a_{k-1}\right|+\frac{k(k-1)}{2}}\left\{m_{(k)}\right\}\left\{a_{1}, \ldots, a_{k}\right\} .
$$

We will also note that

$$
\left|\tilde{m_{(k)}}\right|=\left|m_{(k)}\right|+k-1=\left|m_{(k)}\right|+d\left(m_{(k)}\right) .
$$

The reasoning is amply explained in, say, Getzler and Jones [9] as well as the author's earlier papers. This well-known trick is often employed to make all summands of $m$ odd with respect to the new (suspended) degree, so that $\tilde{m}$ can be manipulated as one entity. For example, in the definition of $A_{\infty}$ algebras in the next subsection, we have $\left|m_{(k)}\right| \equiv k(\bmod 2)$, therefore $\left|\tilde{m}_{(k)}\right|$ is odd.

\subsection{2. $A_{\infty}$ algebras}

We will use the terms " $A_{\infty}$ " and "strongly homotopy associative" interchangeably. Introduced by Stasheff in [24] and [25], and by now a well-known construct, an $A_{\infty}$ algebra structure on a super graded vector space $A$ is defined by a mega operator $m=m_{(1)}+m_{(2)}+\cdots m_{(n)}+\cdots \in \operatorname{Hom}(T A, A)$, with $\left|m_{(k)}\right|$ having the same parity as $k$, and satisfying

$$
\{\tilde{m}\}\{\tilde{m}\}=0 .
$$


This identity is split into sub-identities

$$
\{\tilde{m}\}\{\tilde{m}\}\left\{a_{1}, \ldots, a_{n}\right\}=0 \text { for all } n \geqslant 1,
$$

each of which has finitely many terms, namely,

$$
\sum_{j+k=n+1}\left\{\tilde{m}_{(j)}\right\}\left\{\tilde{m}_{(k)}\right\}\left\{a_{1}, \ldots, a_{n}\right\}=0 .
$$

In $[\mathbf{2}]$, we showed that these are equivalent to

$$
\sum_{j+k=n+1}(-1)^{j}\left\{m_{(j)}\right\}\left\{m_{(k)}\right\}\left\{a_{1}, \ldots, a_{n}\right\}=0 .
$$

Example 3.1. [2] It is easily checked using the last equation that given any associative algebra $A$ with bilinear product $m$, the maps $m_{(n)}: A^{\otimes n} \rightarrow A$, defined by zero if $n$ is odd and the unambiguous $(n-1)$-iterated product if $n$ is even, constitute a nontrivial $A_{\infty}$ structure on $A$.

Stasheff's statement [25] (see also [9], [11]) that an $A_{\infty}$ structure on $A$ corresponds to a square zero coderivation (of degree -1 ) on $T(s A)$ now follows in one easy step: on both sides, the statement amounts to $\{\tilde{m}\}\{\tilde{m}\}=0$ in our notation.

It has been pointed out to us by Stasheff that physicists use the term "weak $A_{\infty}$ " to refer to similar algebras with nonzero element $m_{(0)}$ so that $m_{(1)}$ is no longer square-free; our use of the traditional phrase "weakly homotopy" is not intended to revoke this condition.

\subsubsection{Pre- $L_{\infty}$ and $L_{\infty}$ algebras}

Strongly homotopy Lie algebras were introduced by Schlessinger and Stasheff in [23]. Given an $A_{\infty}$ algebra, one may consider the effects of anti-symmetrizing each map $m_{(n)}$. In [2], we showed that the new structure $\ell=\ell_{(1)}+\ell_{(2)}+\cdots+\ell_{(n)}+\cdots$ satisfies the usual $L_{\infty}$ identities $([\mathbf{1 8}],[\mathbf{1 7}])$ because

$$
\{\tilde{m}\}\{\tilde{m}\}\left\{a_{1}\right\} \cdots\left\{a_{n}\right\}=0
$$

holds for all $n$. Of course, this particular $L_{\infty}$ construction had been in the literature for some time. But then, the new formulation of the $L_{\infty}$ identities led to a simple definition of a pre- $L_{\infty}$ algebra: although a map $m$ need not satisfy the identities

$$
\{\tilde{m}\}\{\tilde{m}\}\left\{a_{1}, \ldots, a_{n}\right\}=0 \text { for all } n,
$$

it is enough to find the right pre- $L_{\infty}$ condition

$$
\{\tilde{m}\}\{\tilde{m}\}\left\{a_{1}, \ldots,\left\{a_{n-1}\right\}\left\{a_{n}\right\}\right\}=0 \text { for all } n
$$

in order to conclude that the anti-symmetrization is $L_{\infty}$ (the additional braces around $a_{n-1}$ and $a_{n}$ indicate, by definition of multibraces, that the signed sum over the permutations of these two elements is taken). For $m=m_{(2)}$ and $n=3$ this is indeed the usual definition of a right pre-Lie algebra. Similarly, one may look for the left pre- $L_{\infty}$ identities

$$
\{\tilde{m}\}\{\tilde{m}\}\left\{\left\{a_{1}\right\}\left\{a_{2}\right\}, \ldots, a_{n}\right\}=0 \text { for all } n,
$$


or any identity of this type which falls short of $A_{\infty}$ but leads to the $L_{\infty}$ condition above (after being summed over permutations).

Example 3.2. The anti-symmetrization of the map in the example of Subsection 3.1.2 will lead to an $L_{\infty}$ algebra structure on any associative algebra.

Example 3.3. [13, 28, 3] The maps $m_{(1)}, m_{(1 \mid 1)}, m_{(1|1| 1)}, \ldots$ of the homotopy $G$-algebra structure described in Section 4 form a pre- $L_{\infty}$ algebra.

Example 3.4. $[1,16]$ The operators $\Phi_{B}^{1}, \Phi_{B}^{2}, \Phi_{B}^{3}, \ldots$ introduced by the author that measure the order of a differential operator $B$ were shown to constitute an $L_{\infty}$ algebra by Kravchenko in case of a super commutative associative base algebra. Here $B$ is an odd, linear, square-zero map that is not necessarily a second-order differential operator.

We note that an $L_{\infty}$ algebra structure on a vector space $A$ corresponds to a square-zero codifferential on the exterior coalgebra $\Lambda(s A)$ (see e.g. [17]).

\subsection{Generic (weakly) homotopy algebras}

Instead of the usual definition of a "weakly homotopy algebra" structure on the underlying vector space $A$ as a set of maps that reduce to a classical structure in the $m(1)$-cohomology, we will adopt one that is given by the almost obvious mega identity

$$
\{\tilde{m}\}\{\tilde{m}, \tilde{m}, \ldots\}=0
$$

where

$$
\tilde{m}=\sum_{\pi} \tilde{m}_{\pi}
$$

Since there are occasional examples where the identity does not hold (such as the TVOA, whose weak $G_{\infty}$ structure is yet to be described), we may want to call the above property a construction, rather than a definition, that routinely gives rise to homotopy algebras. The mega map $m$ is best thought of as a formal sum of partitioned multilinear maps $m_{\pi}$, one for each partition $\pi$, some of which may be identically zero. Then the "algebra of partitions", introduced in $[\mathbf{3}]$ and denoted by $\mathcal{P}$, determines the sub-identities. For every $\pi$, we find all expressions in $\mathcal{P}$ of the form $\pi^{\prime} *\left[\pi_{1}, \ldots, \pi_{k}\right]$, necessarily finitely many, that exhibit $\pi$ as a summand in the final product (partitions have coefficient one or zero in all products of basis elements by definition). These products correspond to the substitutions of partitioned maps in Subsections 2.2.1 and 2.2.2. Then we sum over the compositions modeled after these products, and set the result equal to zero (some degree restrictions apply). For example, the partition $(1 \mid 1)$ occurs as a term only in the partition products $(1) *(1 \mid 1),(1 \mid 1) *(1),(2) *(1 \mid 0)$, and $(2) *(0 \mid 1)$, but nowhere else. Therefore, the sub-identity corresponding to the partition $(1 \mid 1)$ is written as

$$
\left[\left\{m_{(1)}\right\}\left\{m_{(1 \mid 1)}\right\} \pm\left\{m_{(1 \mid 1)}\right\}\left\{m_{(1)}\right\} \pm\left\{m_{(2)}\right\}\left\{m_{(1 \mid 0)}\right\} \pm\left\{m_{(2)}\right\}\left\{m_{(0 \mid 1)}\right\}\right]\{a \mid b\}=0 .
$$

The mega map and the mega identity above were proposed by the author [3] as the "strongly homotopy Gerstenhaber algebra" or " $G_{\infty}$ algebra" structure on a 
super graded vector space, following the terminology of the articles [7], [13], and $[\mathbf{2 8}]$. However, we now propose that this identity be known as a generic "homotopy algebra" structure, whereas the term "(weakly) homotopy $G$ algebra" be reserved for homotopy algebras with $m_{(1 \mid 0)}$ as the identity map that chooses one element from one slot of vectors and nothing from the very next (there may be several ways of choosin' nuttin') and $m_{(0 \mid 1)}$ as the zero map. In particular, the anti-symmetrization of $m_{(1 \mid 1)}$ becomes the G-bracket and the bilinear product $m_{(2)}$ becomes the super commutative associative map of the $G$-algebra. The main reason is the simplicity and all-encompassing quality of the more general identity: almost all structures studied under the homotopy umbrella are defined by identities comprising sums of compositions of multilinear maps (there are NO new identities!). Although we are mostly concerned with compositions of one mega map with itself, the necessity of composing such a map with several copies of itself has naturally arisen (again see $[\mathbf{7}],[\mathbf{1 3}]$, and $[\mathbf{2 8}])$.

Due to its simplicity, the identity easily lends itself to modifications and generalizations. Substructures are easy to identify (examples will follow), symmetries can be arranged at will, and the partition algebra as well as the depth of the compositions (currently two) may be replaced.

A note on the suspended grading: we define [3]

$$
\left|\tilde{m}_{\pi}\right|=d(\pi)+\bar{d}(\pi)+\left|m_{\pi}\right|+1,
$$

at least up to congruence modulo two, where $\bar{d}(\pi)$ denotes the number of slots in the partition minus two. In other words, we add the super degree of $\left|m_{\pi}\right|$ to the number of arguments and slots of $\pi$, and adopt the resulting parity. The $\bar{d}$-degree will resurface in the study of topological vertex operator algebras.

\subsection{Classical and (weakly) homotopy Gerstenhaber algebras}

A (classical) Gerstenhaber algebra is a super graded vector space $A$ with a super commutative, associative bilinear product (suppressed) and an odd bracket $[,]_{G}$ satisfying

(i) $[a, b]_{G}=-(-1)^{(|a|-1)(|b|-1)}[b, a]_{G}$ (antisymmetry with suspended grading),

(ii) $\left[a,[b, c]_{G}\right]_{G}=\left[[a, b]_{G}, c\right]_{G}+(-1)^{(|a|-1)(|b|-1)}\left[b,[a, c]_{G}\right]_{G}$ (Leibniz property with suspended grading), and

(iii) $[a, b c]_{G}=[a, b]_{G} c+(-1)^{(|a|-1)|b|} b[a, c]_{G}$ (Poisson rule with mixed grading).

The name comes from the original construction of Gerstenhaber [6] on the cohomology of the Hochschild algebra of a super commutative associative algebra. Namely, the super anti-symmetrization of the braces \{\}\{\} mentioned earlier, and the "dot product" of multilinear maps, descend to a G-bracket and a commutative associative product with the above properties respectively.

We defined a homotopy Gerstenhaber algebra in Section 3.2 loosely as a homotopy algebra with a specific operator $m_{(1 \mid 0)}$ that makes the cohomology into a commutative associative algebra and therefore can be thought of as an $A_{\infty}$ algebra with additional structure. (There are already such structures in the literature. For example, in [21], Markl mentions "strongly homotopy associative commutative algebras", a.k.a. $C_{\infty}$ or balanced $A_{\infty}$ algebras, citing [20] and [12].) Note that we anti-symmetrize $m_{(1 \mid 1)}$ to obtain a G-bracket in the $m_{(1)}$-cohomology. 
Besides the homotopy G-algebra structure described in Section 3.2, another natural way to define a homotopy Gerstenhaber algebra would be via higher brackets $g=\sum g_{\pi}$ (plus some homotopy G-algebra map $m$ in the first sense, to take the place of the super commutative and associative product), one of which directly descends to a G-bracket on the $m_{(1)}$-cohomology. The brackets need not be antisymmetrized. This not only resonates with the way $L_{\infty}$ algebras are defined, but also happens to be the structure on a topological vertex operator algebra (as a homotopy BV-algebra) as we will see. In this new definition, we may want to use the term homotopy Gerstenhaber bracket algebra, or homotopy GB-algebra, to underline the difference (apart from the switch from "map" to "bracket" in terminology, and the anti-symmetrization issue, the difference will be mostly philosophical). Depending on example, some general identities resembling those of the classical G-algebras may be imposed on $g$ and $m$. We do not have this problem of "looking like a classical algebra" versus "descending to a classical algebra" in $A_{\infty}$ algebras, for example, because these structures both "look like" and "descend to" associative algebras. Speaking of $A_{\infty}$ algebras, it is our observation that maps of plain types commonly arise from some inner structure of a vector space $A$, whereas most partitioned maps behave like substitution operators on some higher structure (like endomorphisms, mixed tensor space, Hochschild complex...) on $A$ and do not refer to the algebra structure on $A$, if any. The Hochschild complex of an associative algebra $(A, m)$ is a good example. The maps of types (1) and (2) are defined in terms of the multiplication $m$ (and also in terms of substitution operators), but maps of partition type $(1 \mid n)$ are substitution operators for multilinear maps on $A$.

Two substructures of homotopy G-algebras $(A, m)$ were mentioned in [3], namely the $A_{\infty}$ subalgebra

$$
m_{(1)}+m_{(2)}+m_{(3)}+\cdots
$$

and the pre- $L_{\infty}$ algebra

$$
m_{(1)}+m_{(1 \mid 1)}+m_{(1|1| 1)}+\cdots .
$$

For the first, the mega identity is reduced to the usual

$$
\{\tilde{m}\}\{\tilde{m}\}=0,
$$

and for the second, it is replaced by the right pre- $L_{\infty}$ condition

$$
\{\tilde{m}\}\{\tilde{m}\}\left\{a_{1}, \ldots,\left\{a_{n-1}\right\}\left\{a_{n}\right\}\right\}=0 \text { for all } n .
$$

\subsection{Higher order differential operators}

The notion of a higher order differential operator $B \in \operatorname{Hom}(A, A)$ for a noncommutative, nonassociative algebra $\left(A, m_{(2)}\right)$ was introduced by the author in [1]. The definition was modeled after Koszul's [15] for commutative, associative algebras. We include the definition for reference $\left(m_{(2)}\right.$ suppressed). Let

$$
\begin{aligned}
\Phi_{B}^{1}(a) & =B(a) \\
\Phi_{B}^{2}(a, b) & =\Phi_{B}^{1}(a b)-\Phi_{B}^{1}(a) b-(-1)^{|B||a|} a B(b)
\end{aligned}
$$




$$
\begin{aligned}
\Phi_{B}^{r+2}\left(a_{1}, \ldots, a_{r}, b, c\right)= & \Phi_{B}^{r+1}\left(a_{1}, \ldots, a_{r}, b c\right)-\Phi_{B}^{r+1}\left(a_{1}, \ldots, a_{r}, b\right) c \\
& -(-1)^{|b|\left(|B|+\left|a_{1}\right|+\cdots+\left|a_{r}\right|\right)} b \Phi_{B}^{r+1}\left(a_{1}, \ldots, a_{r}, c\right), \quad r \geqslant 1 .
\end{aligned}
$$

Each bracket measures the previous one's failure to be a derivation of $m_{(2)}$ in the last argument; then we say $B$ is a differential operator of order (at most) $r$ if $\Phi_{B}^{r+1}$ identically vanishes. For a partitioned-map presentation of the formula see [3]. Later, Kravchenko showed in [16] that the higher brackets $\Phi_{B}^{r}$ form an $L_{\infty}$ algebra structure in case $A$ is super commutative. A generalization of the maps $B$ and $m_{(2)}$ in the $\Phi$-operators to arbitrary multilinear maps is given in [2].

\subsection{Classical and weakly homotopy Batalin-Vilkovisky algebras}

The usual definition of a (classical) Batalin-Vilkovisky algebra (or BV-algebra) is a Gerstenhaber algebra (with a super commutative associative bilinear product $\left.m_{(2)}\right)$ that is obtained by taking the G-bracket of the bilinear map $m_{(2)}$ with an odd, linear map $\beta$, which is also required to be a second order differential operator with respect to $m_{(2)}$. More precisely, we construct

$$
[a, b]_{\beta}=(-1)^{|a|}\left[\beta, m_{(2)}\right]\{a, b\}=(-1)^{|a|} \Phi_{\beta}^{2}(a, b),
$$

where the bracket in the middle is the Gerstenhaber bracket of two multilinear maps, and prove that the result is a classical G-algebra (see $[\mathbf{6}, \mathbf{2}]$ ).

A weakly homotopy Batalin-Vilkovisky algebra (or differential BV-algebra, as it has been called in literature) should be a super graded vector space with a number of higher multilinear maps, in particular an odd, square-zero operator $m_{(1)}$ that is a derivation of some even, bilinear product $m_{(2)}$, and an odd, linear, square-zero operator $B$ that is a second-order differential operator with respect to $m_{(2)}$. We require $m_{(2)}$ be super commutative and associative up to homotopy (although homotopy associativity is needed only to fill out the definition of the classical algebra in the cohomology), and the commutator $\left[B, m_{(1)}\right]$ be a diagonalizable operator $L$ (possibly zero) that commutes with the two degree operators. Under these conditions, it was shown in [1] that all these maps collapse to a classical BV-algebra structure on the $m_{(1)}$-cohomology. (Kosmann-Schwarzbach commented to us that there are examples of homotopy BV-algebras where the commutator $L$ is not diagonalizable.)

We may, and indeed will, define a stronger notion of a homotopy BV-algebra based on the two prominent examples to be discussed at the end of the article. We will require a homotopy G-algebra structure and a map $B$ with all the trimmings described above. (Homotopy G-algebras generalize super commutative associative algebras.) How many classical G-algebra structures does this set-up generate? As we have mentioned above, there is the G-algebra on the $m_{(1)}$-cohomology, coming from the anti-symmetrization of $m_{(1 \mid 1)}$, and also the one induced directly by the bracket $\left[B, m_{(2)}\right]$ on the same space. This is exactly the situation in a topological vertex operator algebra. In the meantime, we will be freely using the G-bracket notation with no indices on the Hochschild complex to denote the usual anti-symmetrization of simple composition. Too many G's? We also use the name "Gerstenhaber" occasionally to refer to a certain mathematician with vast contributions to the field! 
Definition 3.1. A weakly homotopy Batalin-Vilkovisky algebra is a graded vector space A together with a homotopy G-map $m=\sum_{\pi} m_{\pi}$ and an odd, linear, squarezero, second-order differential operator $B$ with respect to $m_{(2)}$ such that the even linear operator

$$
L=\left[B, m_{(1)}\right]
$$

is diagonalizable. As in vertex operator algebra theory, we will call the eigenvalues of $L$ weights, and note that they produce a third grading on the Hochschild complex of $A$, by definition commuting with the other two. (In many examples, $L$ will be zero.)

Example 3.5. A topological vertex operator algebra has a homotopy BV-algebra structure: Subsection 4.2.2.

This definition will give us a higher $B V$ bracket

$$
[,]_{B}^{\pi}=\left[B, m_{\pi}\right]
$$

for every partition $\pi$, collectively giving us a homotopy GB-algebra as a formal sum of brackets that satisfy certain identities, just as an $L_{\infty}$ algebra structure is a sum of brackets satisfying identities.

\section{The homotopy algebra structures of the Hochschild space and topological vertex operator algebra}

\subsection{The homotopy G-algebra structures}

4.1.1. On the Hochschild complex

We will review the information in $[\mathbf{7}]$ and $[\mathbf{3}]$ about the homotopy G-algebra structure on $C^{\bullet}(A, A)$ for an associative algebra $\left(A, m_{(2)}\right)$. The nonzero operators are as follows:

$\left\{M_{(1)}\right\}\{x\}=\left[m_{(2)}, x\right]$

$\left\{M_{(2)}\right\}\{x, y\}= \pm\left\{m_{(2)}\right\}\{x, y\}$

$\left\{M_{(1 \mid 0)}\right\}=$ identity map on the left slot (for symmetry)

$\left\{M_{(1 \mid 1)}\right\}\{x \mid y\}=\{x\}\{y\}$ (shown by Gerstenhaber in [6] to be right pre-Lie; see[2])

$\left\{M_{(1 \mid n)}\right\}\left\{x \mid y_{1}, \ldots, y_{n}\right\}= \pm\{x\}\left\{y_{1}, \ldots, y_{n}\right\}$ for $n \geqslant 1$

Therefore, the mega map $M$ can be written as the infinite sum

$$
M=M_{(1)}+M_{(2)}+M_{(1 \mid 0)}+M_{(1 \mid 1)}+\sum_{n>1} M_{(1 \mid n)},
$$

satisfying the mega identity

$$
\{\tilde{M}\}\{\tilde{M}, \tilde{M}, \ldots\}=0 .
$$

Note that the map $M_{(1 \mid 1)}$ is the composition operator described earlier under "standard composition". The following individual identities were observed by Gerstenhaber and Voronov in [7] and rewritten in the following form in [3]:

(i) $M_{(1)}^{2}=0$

(ii) $\left(\left\{M_{(1)}\right\}\left\{M_{(2)}\right\} \pm\left\{M_{(2)}\right\}\left\{M_{(1)}\right\}\right)(2)=0$

(iii) $\left(\left\{M_{(2)}\right\}\left\{M_{(2)}\right\}\right)(3)=0$ 


$$
\begin{aligned}
& \text { (iv) }\left(\left\{M_{(1)}\right\}\left\{M_{(1 \mid n+1)}\right\} \pm\left\{M_{(1 \mid n+1)}\right\}\left\{M_{(1)}\right\}\right. \\
& \left. \pm\left\{M_{(2)}\right\}\left\{M_{(1 \mid n)}\right\} \pm\left\{M_{(1 \mid n)}\right\}\left\{M_{(2)}\right\}\right)(1 \mid n+1)=0(n \geqslant 1) \\
& \text { (v) }\left(\left\{M_{(2)}\right\}\left\{M_{(1 \mid n)}\right\} \pm\left\{M_{(1 \mid n)}\right\}\left\{M_{(2)}\right\}\right. \\
& \left.+\sum_{1 \leqslant i \leqslant n-1} \pm\left\{M_{(2)}\right\}\left\{M_{(1 \mid i)}, M_{(1 \mid n-i)}\right\}\right)(2 \mid n)=0
\end{aligned}
$$

(vi) $\left(\left\{M_{(1)}\right\}\left\{M_{(1 \mid 1)}\right\} \pm\left\{M_{(1 \mid 1)}\right\}\left\{M_{(1)}\right\} \pm\left\{M_{(2)}\right\}\left\{M_{(1 \mid 0)}\right\}\right)(1 \mid 1)=0$

One powerful example of the efficiency of the partitioned-braces language is the newly acquired meaning of the formerly stray identity (v). This represents the subidentity corresponding to the partition $(2 \mid n)$, and has terms arising from the compositions of all partitioned maps whose composites contain multilinear maps of type $(2 \mid n)$. Note the substitution of two $M$ 's into one as a change from the previous examples. This is in fact why the author decided to try a different mega identity from that of $A_{\infty}$ in [3]. If substitutions of more complicated types arise in examples in the future, we will easily amend the mega-identity, now that we know more of the mechanism behind it.

\subsubsection{On a TVOA}

The homotopy G-algebra structure on a TVOA was revealed by Kimura, Voronov, and Zuckerman in $[\mathbf{1 3}, \mathbf{2 8}]$, albeit without any explicitly constructed maps. (For a complete set of defining properties of a TVOA and some that follow from the definition, see Appendix B.) The pictures and the lower identities in [13] were translated into the mega identity $\{\tilde{m}\}\{\tilde{m}, \tilde{m}, \ldots\}=0$ in $[\mathbf{3}]$. The similarities between Lian and Zuckerman's earlier explicit maps and relations [19] of homotopy-BV type and the ones studied by Kimura et al. led the author to seek the connections between the two structures and wonder which, if either, was the fundamental one.

The operators constructed in [13] were of all partition types with no zeros, $m_{(1 \mid 0)}$ being the single exception. After Voronov's corrections in [28], it turned out that our generic homotopy algebra mega-identity was an inadequate model for the particular weakly homotopy G-algebra structure on TVOA's. Namely, only the infinitely many identities that correspond to partitions $\pi$ where there is no zero slot, and at most two slots are greater than or equal to two, survived. Unfortunately, such maps plus $m_{(1 \mid 0)}$ do not form a G-substructure, solely because of $m_{(1 \mid 0)}$ (maps of forbidden partition types appear in valid identities). A compact algebraic description of the whole weakly homotopy G-algebra structure on a TVOA is so far nonexistent.

In [13], multibraces denote the equivalent of our partitioned maps. In order to preserve the useful braces notation from earlier work, and to emphasize the importance of the partitions, we have changed the notation of Kimura et al.

\subsection{The homotopy BV-algebra structures}

\subsubsection{On the Hochschild complex}

We will prove in general that any weakly homotopy algebra structure on $A$, including that of a homotopy BV-algebra, can be lifted naturally to the Hochschild complex, by simplifying Getzler's construction in $[\mathbf{8}]$ for $A_{\infty}$ algebras (see also the interpretation in $[\mathbf{2}]$, p.157). 
Theorem 4.1. Let $(A, m)$ be a weakly homotopy algebra (possibly with an additional $B V$ operator $\beta)$. Then its structure can be lifted to the Hochschild complex $C^{\bullet}(A, A)$ by defining

$$
\left\{M_{\pi}\right\}^{\prime}\left\{\left\{x^{\pi}\right\}\right\}^{\prime}=\left\{\left\{m_{\pi}\right\}\left\{x^{\pi}\right\}\right\}^{\prime} .
$$

In case of a $B V$ operator $\beta$ on $A$, we define a $B V$ operator $B$ on $C \bullet(A, A)$ by

$$
\{B\}^{\prime}\{\{x\}\}^{\prime}=\{\{\beta\}\{x\}\}^{\prime} .
$$

Proof. With the braces notation, there really isn't much to prove.

$$
\begin{aligned}
& \{M\}^{\prime}\{M, M, M, \ldots\}^{\prime}\left\{\left\{x^{\pi}\right\}\right\}^{\prime} \\
= & \sum_{\text {product }}=\pi \\
= & \sum_{\text {product }}\{\pi \\
= & \left\{\left\{M_{\pi^{\prime}}\right\}^{\prime}\left\{m_{\pi_{1}^{\prime}}, M_{\pi_{2}^{\prime}}, M_{\pi_{3}^{\prime}}, \ldots\right\}^{\prime}\left\{\left\{m_{\pi_{1}^{\prime}}, m_{\pi_{2}^{\prime}}, m_{\pi_{3}^{\prime}}, \ldots\right\}\left\{x^{\pi}\right\}\right\}^{\prime}\right. \\
= & 0 .
\end{aligned}
$$

Clearly, $B$ is odd and square-zero if $\beta$ is. Finally, we can easily show that

$$
\left\{\Phi_{B}^{3}\right\}^{\prime}\{\{x, y, z\}\}^{\prime}=\left\{\left\{\Phi_{\beta}^{3}\right\}\{x, y, z\}\right\}^{\prime}=0,
$$

that is, $B$ is also a second order differential operator (the first $\phi$ operator is constructed with respect to $M_{(2)}$, and the second one with respect to $\left.m_{(2)}\right)$.

Our construction differs from that of Getzler in that we did not define $M_{(1)}$ by

$$
\left\{M_{(1)}\right\}^{\prime}\{\{x\}\}^{\prime}=\{[m, x]\}^{\prime},
$$

because it would not have worked: the identity $\{m\}\{m\}=0$ (or the $\tilde{m}$ version) is not valid in general, and we would have needed it to prove that $M_{(1)}$ is square-zero.

\subsubsection{On a TVOA}

The observation of Lian and Zuckerman [19] (also see [22]) that a homotopy BatalinVilkovisky structure exists in the cohomology of a topological vertex operator algebra (see Appendix B for full description) predates the discovery of a certain homotopy G-structure on the TVOA itself $([\mathbf{1 3}, \mathbf{2 8}, \mathbf{3}])$. We will put forth strong evidence, with the help of the unifying braces notation, that the former is in fact a consequence of the latter in spite of the chronology. In particular, the homotopy $\mathrm{BV}$-algebra structure on a TVOA, maps, relations, and all, is obtained from the homotopy G-algebra structure by G-bracketing (in the sense of "anti-symmetrization of composition") all maps and relations with an odd, square-zero, second-order differential operator $B$. Again, what is the meaning of a higher order differential operator on a noncommutative, nonassociative algebra? In [1] , we were able to define higher order differential operators in the most general fashion, and show that "half" of the modes $u_{n}$ for each vertex operator $u(z)$ constituted a sequence of differential operators on the TVOA of orders (at most) $1,2,3, \ldots$, while the rest satisfied the properties of multiplication operators. Under this novel definition, the mode $b_{0}$ 
utilized in $[\mathbf{1 9}]$ as the BV operator turned out to be a second-order differential operator with respect to the Wick product, resulting in the vanishing of the higher bracket $\Phi_{b_{0}}^{3}(u, v, t)$. We then defined "generalized" and "differential" BV algebras, on the strength of the existence of second-order differential operators, and gave several examples.

Theorem 4.2. All explicit multilinear maps on a TVOA described by Lian and Zuckerman [19] as part of the homotopy BV algebra structure are either part of a certain weakly homotopy $G$ algebra structure or else can be obtained by taking the $G$-bracket (composition bracket) of $b_{0}$ with homotopy $G$ algebra maps.

Dictionary of maps. The following table exhibits the multilinear operators listed in the Lian-Zuckerman article [19] versus our interpretation of them with $100 \%$ hindsight.

\begin{tabular}{|l|l|}
\hline Lian and Zuckerman call it... & In our language, up to $\pm \ldots$ \\
\hline \hline$b_{0}$ & $B$ \\
\hline$Q$ (BRST differential) & $m_{(1)}$ \\
$\cdot($ dot product, Wick product) & $m_{(2)}$ \\
$n=n(u, v, t)$ & $m_{(3)}$ \\
$m=m(u, v)$ & $m_{(1 \mid 1)}$ \\
\hline$\left[b_{0}, Q\right]=L_{0}$ & {$\left[B, m_{(1)}\right]=L$} \\
$\{\} \quad,[$ BV bracket $)$ & {$\left[B, m_{(2)}\right]=[,]_{B}$} \\
$?$ & {$\left[B, m_{(3)}\right]$} \\
$m^{\prime}=m^{\prime}(u, v)$ & {$\left[B, m_{(1 \mid 1)}\right]$} \\
$n^{\prime \prime}=n^{\prime \prime}(u, v, t) \quad$ appears in Poisson-type identity) & {$\left[m_{(2)},\left[B, m_{(1 \mid 1)}\right]\right]$} \\
\hline
\end{tabular}

For the rest of this subsection we will mostly write maps and identities in our own symbols. It is to be understood that "homotopy G-algebra" in this subsection simply means "weakly homotopy G-algebra" and nothing else. As we have remarked several times earlier, there is no good explicit, algebraic definition of the complete structure on a TVOA, and the generic homotopy algebra identity proposed in this paper is not valid because of deficiencies in higher maps.

\section{Identities or definitions that were known before Lian-Zuckerman.}

1. $\left[m_{(1)}, m_{(2)}\right]=0$ ( $Q$ is a derivation of the Wick, or normal-ordered, product)

2. $\left\{m_{(1)}\right\}^{2}=0\left(Q^{2}=0\right)$

3. $B^{2}=0\left(b_{0}^{2}=0\right)$

4. $L=\left[B, m_{(1)}\right]$ (the commutator of $b_{0}$ with $Q$ is the (Virasoro) degree operator $L_{0}$, commuting with all homogeneous maps)

Explicit maps/identities found by Lian-Zuckerman. For simplicity we will not always specify the sign changes due to crossing of symbols.

5. $\left\{m_{(2)}\right\}^{\text {sym }}=\left\{m_{(2)}\right\}\left\{m_{(1 \mid 0)}\right\}=\left[m_{(1)}, m_{(1 \mid 1)}\right]$

Fundamental homotopy G-identity: Wick is super commutative up to homotopy. First explicit appearance in literature was in [19].

6. $\left\{m_{(2)}\right\}\left\{m_{(2)}\right\} \pm\left[m_{(1)}, m_{(3)}\right]=0$

Fundamental homotopy G-identity: Wick is associative up to homotopy. First explicit appearance in literature again in [19]. 
7. $\left[m_{(1)},\left[B, m_{(2)}\right]\right]=0$

$Q$ is a derivation of the BV-bracket. Why?

$$
\begin{aligned}
& {\left[m_{(1)},\left[B, m_{(2)}\right]\right] } \\
= & {\left[\left[m_{(1)}, B\right], m_{(2)}\right] \pm\left[B,\left[m_{(1)}, m_{(2)}\right]\right] } \\
= & {\left[L, m_{(2)}\right] \pm[B, 0]=0 . }
\end{aligned}
$$

8. $[,]_{B}^{\text {sym }}=\left[B, m_{(2)}\right]^{\text {sym }}=\left[m_{(1)},\left[B, m_{(1 \mid 1)}\right]\right]$

The BV-bracket is symmetric up to homotopy. Can be obtained as follows:

$$
\begin{aligned}
& {\left[m_{(1)},\left[B, m_{(1 \mid 1)}\right]\right] } \\
= & {\left[\left[m_{(1)}, B\right], m_{(1 \mid 1)}\right] \pm\left[B,\left[m_{(1)}, m_{(1 \mid 1)}\right]\right] } \\
= & {\left[L, m_{(1 \mid 1)}\right] \pm\left[B, m_{(2)}^{\text {sym }}\right] } \\
= & \pm\left[B, m_{(2)}\right]^{\text {sym }} .
\end{aligned}
$$

9. $\left[u,[v, t]_{B}\right]_{B}=\left[[u, v]_{B}, t\right]_{B} \pm\left[v,[u, t]_{B}\right]_{B}$

Leibniz property. In $[\mathbf{2}]$ (pp. 154-155), we showed that this is equivalent to the vanishing of the operator

$$
\Phi_{B^{2}}^{3}-\left[B, \Phi_{B}^{3}\right] .
$$

Since $B$ is a square-zero, second-order differential operator, we are done.

10. $\left[u,\left\{m_{(2)}\right\}\{v, t\}\right]_{B}=\left\{m_{(2)}\right\}\left\{[u, v]_{B}, t\right\} \pm\left\{m_{(2)}\right\}\left\{v,[u, t]_{B}\right\}$

Poisson property for left BV-bracketing with respect to the Wick product. Again in $[2]$ (p.155) we showed that this identity is exactly the condition for the operator $\Phi_{B}^{3}$ to be identically zero.

11. $\left[\left\{m_{(2)}\right\}\{u, v\}, t\right]_{B}=\left\{m_{(2)}\right\}\left\{u,[v, t]_{B}\right\} \pm\left\{m_{(2)}\right\}\left\{[u, t]_{B}, v\right\}$ $\pm\left[m_{(1)},\left[m_{(2)},\left[B, m_{(1 \mid 1)}\right]\right]\right]\{u, v, t\}$

Poisson property for right BV-bracketing up to homotopy. We do not have a precooked identity that corresponds to this one (due to the lopsided way we have defined our $\Phi$-operators).

Two more related identities.

12. $\left\{m_{(2)}\right\}\{\{u\}\{v\}, t\}=0$

Found in [3]: Wick is left pre-Lie, hence its G-bracket is Lie. The Lie property of the G-bracket was also in dependently observed by Dong, Li, and Mason in [5].

13. $\left[B,\left\{m_{(2)}\right\}\left\{m_{(2)}\right\}\right]=\left[B,\left[m_{(1)}, m_{(3)}\right]\right]=\left[m_{(1)},\left[B, m_{(3)}\right]\right]$

This identity is obtained by B-bracketing the homotopy associativity statement, and could easily have been discovered by Lian and Zuckerman. The operator $\left[B, m_{(3)}\right]$ was similarly within their scope.

One more homotopy BV structure. By the Theorem in Subsection 4.2.1, there is also a homotopy BV-algebra structure on the Hochschild complex defined on any TVOA.

\subsubsection{Explicit construction?}

How do we construct an explicit homotopy G-map $m$ on a generic TVOA, now that the mystery behind its homotopy BV structure is explained? The key may be found 
in Lian and Zuckerman's construction of the " $B V_{\infty}$ " maps in [19], using the relation

$$
Q b_{-1}+b_{-1} Q=L_{-1}
$$

several times. We will show alternative brute-force constructions of the same maps based on the VOA properties displayed in Appendix B and especially utilizing the important identity above. (We urge the reader to consult the Appendix for notation.) This work is yet unfinished, but completion may become technically possible, due to the existence of an induction argument. The induction is on the $d+\bar{d}-$ degree of the multilinear maps we are to construct (that is, the sum of the numbers of arguments and slots minus three). This particular grading is not preserved under composition; in fact, $d$ is preserved but $\bar{d}$ is decreased by one. With the exception of (1) every partition has a nonnegative $d+\bar{d}$-degree, and the lower maps are already known (see the nontrivial ones below). Now we always have the terms

$$
\left\{m_{(1)}\right\}\left\{m_{\pi^{\prime}}\right\} \pm\left\{m_{\pi^{\prime}}\right\}\left\{m_{(1)}\right\}
$$

in the sub-identity corresponding to type $\pi^{\prime}$. Since $(d+\bar{d})\left(m_{(1)}\right)=-1$, we observe that the $d+\bar{d}$-degree of $m_{\pi^{\prime}}$ (say $n$ ) is one more than the sum of the two $d+\bar{d}$ degrees in the remaining terms (total $n-1$ ) of the sub-identity, or at least one more than the $d+\bar{d}$-degree of every remaining map (at most $n-1$ ). That is, all the other partitioned maps that appear will have been constructed prior to our $m_{\pi^{\prime}}$ ! Then we start with the terms in the rest of the identity and try to put their sum into the form $\left\{m_{(1)}\right\}\left\{m_{\pi^{\prime}}\right\} \pm\left\{m_{\pi^{\prime}}\right\}\left\{m_{(1)}\right\}$.

First, let us investigate the "G-bracket" map $m_{(1 \mid 1)}$. It appears in the sub-identity

$$
\left\{\left\{m_{(1)}\right\}\left\{m_{(1 \mid 1)}\right\} \pm\left\{m_{(1 \mid 1)}\right\}\left\{m_{(1)}\right\} \pm\left\{m_{(2)}\right\}\left\{m_{(1 \mid 0)}\right\}\right\}\{u \mid v\}=0 .
$$

We then start with the last term and note that, after cancelations, the expression is in the image of the "differentiation" operator $L_{-1}=Q b_{-1}+b_{-1} Q$ :

$$
\begin{aligned}
& \left\{m_{(2)}\right\}\left\{m_{(1 \mid 0)}\right\}\{u \mid v\} \\
= & u_{-1} v-v_{-1} u \\
= & {\left[u_{-1}, v_{-1}\right] \mathbf{1} } \\
= & \sum_{i \geqslant 0}(-1)^{i}\left(u_{i} v\right)_{-2-i} \mathbf{1} \\
= & \sum_{i \geqslant 0}(-1)^{i} \frac{1}{-1-i}\left[L_{-1},\left(u_{i} v\right)_{-1-i}\right] \mathbf{1} \\
= & \sum_{i \geqslant 0} \frac{(-1)^{i+1}}{i+1} L_{-1}\left(u_{i} v\right)_{-1-i} \mathbf{1} \\
= & \sum_{i \geqslant 0} \frac{(-1)^{i+1}}{i+1}\left(Q b_{-1}+b_{-1} Q\right)\left(u_{i} v\right)_{-1-i} \mathbf{1} \\
= & Q\left(\sum_{i \geqslant 0} \frac{(-1)^{i+1}}{i+1} b_{-1}\left(u_{i} v\right)_{-1-i} \mathbf{1}\right)
\end{aligned}
$$


Homology, Homotopy and Applications, vol. 7(2), 2005

$$
\begin{aligned}
& +\sum_{i \geqslant 0} \frac{(-1)^{i+1}}{i+1} b_{-1}\left((Q u)_{i} v\right)_{-1-i} \mathbf{1} \pm \sum_{i \geqslant 0} \frac{(-1)^{i+1}}{i+1} b_{-1}\left(u_{i}(Q v)\right)_{-1-i} \mathbf{1} \\
= & {\left[Q, m_{(1 \mid 1)}\right]\{u \mid v\}, }
\end{aligned}
$$

where

$$
\left\{m_{(1 \mid 1)}\right\}\{u \mid v\}=\sum_{i \geqslant 0} \frac{(-1)^{i+1}}{i+1} b_{-1}\left(u_{i} v\right)_{-1-i} \mathbf{1}=\sum_{i \geqslant 0} \frac{(-1)^{i+1}}{i+1}\left(b_{-1} u_{i} v\right)_{-1-i} \mathbf{1} .
$$

Similarly, the partition (3) gives rise to the identity

$$
\left\{\left\{m_{(1)}\right\}\left\{m_{(3)}\right\} \pm\left\{m_{(3)}\right\}\left\{m_{(1)}\right\} \pm\left\{m_{(2)}\right\}\left\{m_{(2)}\right\}\right\}\{u, v, w\}=0 .
$$

We start again from the term that has already been defined, i.e.

$$
\begin{aligned}
& \left\{m_{(2)}\right\}\left\{m_{(2)}\right\}\{u, v, w\} \\
= & \left(u_{-1} v\right)_{-1} w-u_{-1}\left(v_{-1} w\right) \\
= & \left(\sum_{i \geqslant 0} u_{-1+i} v_{-1-i}+v_{-2-i} u_{i}\right) w-u_{-1} v_{-1} w \\
= & \sum_{i \geqslant 1} u_{-1+i} v_{-1-i} w+\sum_{i \geqslant 0} v_{-2-i} u_{i} w \\
= & \sum_{i \geqslant 0} u_{i} v_{-2-i} w+\sum_{i \geqslant 0} v_{-2-i} u_{i} w \\
= & \sum_{i \geqslant 0} \frac{1}{i+1}\left(L_{-1} u\right)_{i+1} v_{-i-2} w+\sum_{i \geqslant 0} \frac{1}{-i-1}\left(L_{-1} v\right)_{-i-1} u_{i} w \\
= & \sum_{i \geqslant 0} \frac{1}{i+1}\left(\left(Q b_{-1}+b_{-1} Q\right) u\right)_{i+1} v_{-i-2} w+\sum_{i \geqslant 0} \frac{1}{-i-1}\left(\left(Q b_{-1}+b_{-1} Q\right) v\right)_{-i-1} u_{i} w \\
= & \sum_{i \geqslant 0} \frac{1}{i+1}\left[Q,\left(b_{-1} u\right)_{i+1}\right] v_{-i-2} w+\sum_{i \geqslant 0} \frac{1}{i+1}\left(b_{-1} Q u\right)_{i+1} v_{-i-2} w \\
& -\sum_{i \geqslant 0} \frac{1}{i+1}\left[Q,\left(b_{-1} v\right)_{-i-1}\right] u_{i} v-\sum_{i \geqslant 0} \frac{1}{i+1}\left(b_{-1} Q v\right)_{-i-1} u_{i} w \\
= & \sum_{i \geqslant 0} \frac{1}{i+1} Q\left(b_{-1} u\right)_{i+1} v_{-i-2} w \pm \sum_{i \geqslant 0} \frac{1}{i+1}\left(b_{-1} u\right)_{i+1} Q v_{-i-2} w \\
& \pm \sum_{i \geqslant 0} \frac{1}{i+1}\left(b_{-1} Q u\right)_{i+1} v_{-i-2} w-\sum_{i \geqslant 0} \frac{1}{i+1} Q\left(b_{-1} v\right)_{-i-1} u_{i} w \\
& \pm \sum_{i \geqslant 0} \frac{1}{i+1}\left(b_{-1} v\right)_{-i-1} Q u_{i} w \pm \sum_{i \geqslant 0} \frac{1}{i+1}\left(b_{-1} Q v\right)_{-i-1} u_{i} w \\
= & \{Q\}\left\{m_{(3)}\right\}\{u, v, w\} \pm\left\{m_{(3)}\right\}\{Q\}\{u, v, w\}, \\
& =1
\end{aligned}
$$

with

$$
\left\{m_{(3)}\right\}\{u, v, w\}=\sum_{i \geqslant 0} \frac{1}{i+1}\left(b_{-1} u\right)_{i+1} v_{-i-2} w-\sum_{i \geqslant 0} \frac{1}{i+1}\left(b_{-1} v\right)_{-i-1} u_{i} w .
$$


In order to extend the construction to $m$, it remains to show that every "computed expression" that we start with is in fact in the image of $L_{-1}$. Or, we need to ascertain that every such expression starts with some mode $u_{n}$ with $n \neq-1$, as we have

$$
u_{n}=\frac{1}{n+1}\left[L_{-1}, u_{n+1}\right]
$$

We would like to emphasize that the existence and the types of these maps (if they do exist) have not been determined.

\section{Summary of proposed classification}

We have defined a general weakly homotopy algebra on a super graded vector space $A$ by the relation

$$
\{\tilde{m}\}\{\tilde{m}, \tilde{m}, \ldots\}=0
$$

where

$$
m=\sum_{\pi} m_{\pi} \in C_{p a r}^{\bullet}(A, A),
$$

and there are no conditions on the partitioned maps $m_{\pi}$ except possibly for the super degrees. In order to obtain a super commutative, associative algebra in the $m_{(1)}$-cohomology, we impose the restriction

$$
m_{(1 \mid 0)}=\text { identity and } m_{(0 \mid 1)}=\text { zero }
$$

on the homotopy algebra and obtain a weakly homotopy Gerstenhaber algebra. Most explicit examples discussed in this article fall into this class. If there is a homotopy G-algebra structure on $A$ as well as an odd, linear, square-zero, secondorder differential operator $B$ (with respect to the bilinear map $m_{(2)}$ ), then we call the resulting form a weakly homotopy Batalin-Vilkovisky algebra. But since $G$-bracketing $m$ with $B$ yields a sum $g=\sum[,]_{B}^{\pi}$ of higher BV brackets on $A$, descending to a classical BV algebra bracket on the cohomology, we also introduce the concept of a weakly homotopy Gerstenhaber bracket algebra having a homotopy G-map $m$ and a bracket map $g$. The classical G-algebra structure that exists on the cohomology in both cases is induced by the anti-symmetrization of $m_{(1 \mid 1)}$ and the product $m_{(2)}$, but in the second case, we also have the G-bracket $g_{(2)}$ or the $\mathrm{BV}$-algebra bracket $\left[B, m_{(2)}\right]$ that descends to the cohomology, together with $m_{(2)}$.

A strongly homotopy associative algebra $\left(A_{\infty}\right.$ algebra $)$ is a homotopy algebra with only nonzero maps of partition type $(n)$, satisfying

$$
\{\tilde{m}\}\{\tilde{m}\}=0,
$$

and its anti-symmetrization leads to an $L_{\infty}$ algebra, or strongly homotopy Lie algebra, of brackets. In fact, any algebra $(A, m)$ that produces $L_{\infty}$ under antisymmetrization may be called pre- $L_{\infty}$ and may possibly satisfy

$$
\{\tilde{m}\}\{\tilde{m}\}\left\{a_{1}, \ldots, a_{n-2},\left\{a_{n-1}\right\}\left\{a_{n}\right\}\right\}=0 \quad \text { or } \quad\{\tilde{m}\}\{\tilde{m}\}\left\{\left\{a_{1}\right\}\left\{a_{2}\right\}, \ldots, a_{n}\right\}=0
$$


(the right and left pre- $L_{\infty}$ identities respectively). An $L_{\infty}$ algebra obtained by antisymmetrization of some $m$ satisfies the relation

$$
\{\tilde{m}\}\{\tilde{m}\}\left\{a_{1}\right\} \ldots\left\{a_{n}\right\}=0 .
$$

We hope to bring about a new wave of discussions of names and definitions, even though our nomenclature may not be accepted as is.

Acknowledgments. I am indebted to Yvette Kosmann-Schwarzbach, Martin Markl, Jim Stasheff, Sasha Voronov, and the referees for numerous helpful comments and corrections. Any remaining errors are mine, as the saying goes.

\section{Appendix A: Expansion of a partitioned substitution}

In order to understand the mechanics of the generic substitution

$$
\left\{M_{\pi}\right\}\left\{M_{\pi_{1}}^{(1)}, \ldots, M_{\pi_{l}}^{(l)}\right\}\left\{a_{1}^{(1)} \cdots a_{n_{1}}^{(1)}|\cdots| a_{1}^{(k)} \cdots a_{n_{k}}^{(k)}\right\},
$$

we first consider the simplest case of

$$
\left\{M_{(n)}\right\}\left\{N_{\left(i_{1}|\cdots| i_{k}\right)}\right\}\left\{a_{1}^{(1)} \cdots a_{n_{1}}^{(1)}|\cdots| a_{1}^{(k)} \cdots a_{n_{k}}^{(k)}\right\} .
$$

That is, only one partitioned map is to be substituted into $M_{(n)}$ (hence $l=1$ ), and the number of slots in the partition defining the substitution properties of this inner map matches that of the partitioning of the vectors, namely $k$. If each slot of $N_{\left(i_{1}|\cdots| i_{k}\right)}$ is at most equal to the number of vectors in the corresponding slot of the ordered vector set, then we are in business (otherwise substitution is not possible). In the notation of the last Eq., we want $i_{j} \leqslant n_{j}$ for all $1 \leqslant j \leqslant k$.

Example 5.1. Let us start working on an example that we will keep revisiting throughout this Appendix. Consider the substitution

$$
\left\{M_{(4)}\right\}\left\{N_{(1 \mid 3)}\right\}\left\{a_{1}, a_{2}, a_{3} \mid b_{1}, b_{2}, b_{3}, b_{4}\right\} .
$$

Both the middle map and the set of arguments sport two slots $(k=2)$, and in each vector slot there are more vectors than $N_{(1 \mid 3)}$ can hold.

In the general case, we want to split each slot of vectors into three pieces, some of which may be $\emptyset$, in all possible ways. The only condition is that the middle piece be as long as the number in the corresponding slot for $\left\{m_{\left(i_{1}|\cdots| i_{k}\right)}\right\}$. That is, we are getting ready to plug in the middle set in each slot into its designated place inside $\left\{N_{\left(i_{1}|\cdots| i_{k}\right)}\right\}$. We may denote this splitting process by

$$
\begin{gathered}
\left\{a^{(1)}\right\}=\left\{b^{(1)}, c^{(1)}, d^{(1)}\right\} \\
\vdots \\
\left\{a^{(k)}\right\}=\left\{b^{(k)}, c^{(k)}, d^{(k)}\right\},
\end{gathered}
$$

where letters with superscripts only denote blocks of consecutive vectors. In particular, the $c$ 's have the exact number of vectors to fit in the corresponding slot of $m_{\left(i_{1}|\cdots| i_{k}\right)}$. 
Example 5.2. The first slot of vectors in the previous example can be split as

$$
\emptyset\left\{a_{1}\right\} \quad\left\{a_{2}, a_{3}\right\} \text { or }\left\{a_{1}\right\} \quad\left\{a_{2}\right\} \quad\left\{a_{3}\right\} \text { or }\left\{a_{1}, a_{2}\right\} \quad\left\{a_{3}\right\} \quad \emptyset .
$$

Similarly, the second slot offers the possibilities

$$
\emptyset\left\{b_{1}, b_{2}, b_{3}\right\} \quad\left\{b_{4}\right\} \text { and }\left\{b_{1}\right\} \quad\left\{b_{2}, b_{3}, b_{4}\right\} \quad \emptyset .
$$

Therefore, there are altogether six different ways (splittings) in which we may choose to apply $N_{(1 \mid 3)}$ to the partitioned vector set.

The next step is, for each overall choice of splittings, to permute the "left leftovers" in all slots of vectors in such a way that inner orders are preserved. In the same manner we permute all "right leftovers" so that their inner orders are preserved, and apply $\left\{m_{\left(i_{1}|\cdots| i_{k}\right)}\right\}$ to the $c$ 's in such a way that the left leftovers precede, and the right leftovers follow, the output vector (inside $m_{(n)}$ ). After forming the signed sum that reflects all possible permutations, we go ahead and follow the same procedure for all of the other possible splittings.

Example 5.3. For example, the splitting

$$
\left\{a_{1}\right\} \quad\left\{a_{2}\right\} \quad\left\{a_{3}\right\} \quad \mid \quad\left\{b_{1}\right\} \quad\left\{b_{2}, b_{3}, b_{4}\right\} \quad \emptyset
$$

gives rise to the following two terms,

$$
\pm M_{(4)}\left(a_{1}, b_{1}, N_{(1 \mid 3)}\left(a_{2} \mid b_{2}, b_{3}, b_{4}\right), a_{3}\right)
$$

and

$$
\pm M_{(4)}\left(b_{1}, a_{1}, N_{(1 \mid 3)}\left(a_{2} \mid b_{2}, b_{3}, b_{4}\right), a_{3}\right) .
$$

Note that the inner order of the $a$ 's and the b's are preserved. In order to complete the substitution

$$
\left\{M_{(4)}\right\}\left\{N_{(1 \mid 3)}\right\}\left\{a_{1}, a_{2}, a_{3} \mid b_{1}, b_{2}, b_{3}, b_{4}\right\},
$$

the summands coming from each of the remaining five splittings need to be added.

Example 5.4. This is the expansion promised in the second example of Subsection 2.2.2. We have

$$
\begin{aligned}
& \left\{X_{(2 \mid 4)}\right\}\left\{Y_{(1 \mid 2)}\right\}\{a, b|c, d| e, f, g, h\} \\
= & \pm X_{(2 \mid 4)}\left(Y_{(1 \mid 2)}(a \mid c, d), b \mid e, f, g, h\right) \pm X_{(2 \mid 4)}\left(a, Y_{(1 \mid 2)}(b \mid c, d) \mid e, f, g, h\right) \\
& \pm X_{(2 \mid 4)}\left(a, b \mid Y_{(1 \mid 2)}(c \mid e, f), d, g, h\right) \pm X_{(2 \mid 4)}\left(a, b \mid Y_{(1 \mid 2)}(c \mid e, f), g, d, h\right) \\
& \pm X_{(2 \mid 4)}\left(a, b \mid Y_{(1 \mid 2)}(c \mid e, f), g, h, d\right) \pm X_{(2 \mid 4)}\left(a, b \mid e, Y_{(1 \mid 2)}(c \mid f, g), d, h\right) \\
& \pm X_{(2 \mid 4)}\left(a, b \mid e, Y_{(1 \mid 2)}(c \mid f, g), h, d\right) \pm X_{(2 \mid 4)}\left(a, b \mid e, f, Y_{(1 \mid 2)}(c \mid g, h), d\right) \\
& \pm X_{(2 \mid 4)}\left(a, b \mid c, Y_{(1 \mid 2)}(d \mid e, f), g, h\right) \pm X_{(2 \mid 4)}\left(a, b \mid c, e, Y_{(1 \mid 2)}(d \mid f, g), h\right) \\
& \pm X_{(2 \mid 4)}\left(a, b \mid e, c, Y_{(1 \mid 2)}(d \mid f, g), h\right) \pm X_{(2 \mid 4)}\left(a, b \mid c, e, f, Y_{(1 \mid 2)}(d \mid g, h)\right) \\
& \pm X_{(2 \mid 4)}\left(a, b \mid e, c, f, Y_{(1 \mid 2)}(d \mid g, h)\right) \pm X_{(2 \mid 4)}\left(a, b \mid e, f, c, Y_{(1 \mid 2)}(d \mid g, h)\right) .
\end{aligned}
$$

The number of terms in a certain composition is easy to compute. We assume that the set of tri-partitions

$$
\left\{u_{1}, i_{1}, v_{1}\right\}, \ldots,\left\{u_{k}, i_{k}, v_{k}\right\}
$$


of $n_{1}, \ldots, n_{k}$ given above is fixed, where $k \geqslant 1$ and all numbers are nonnegative integers. First let us find the number of permutations of left (or right) leftovers.

Lemma 5.1. Let $S_{1}, \ldots, S_{k}$ be ordered sets with $u_{1}, \ldots, u_{k}$ elements respectively, with $k \geqslant 1$, and $u_{j} \geqslant 0$ for all $1 \leqslant j \leqslant k$. Then the number of permutations of the elements of $S_{1} \cup \cdots \cup S_{k}$ that preserve the inner order of each $S_{j}$ (called unshuffles) is given by the product

$$
\left(\begin{array}{c}
u_{1}+\cdots+u_{k} \\
u_{1}
\end{array}\right)\left(\begin{array}{c}
u_{2}+\cdots+u_{k} \\
u_{2}
\end{array}\right) \cdots\left(\begin{array}{c}
u_{k-1}+u_{k} \\
u_{k-1}
\end{array}\right)\left(\begin{array}{l}
u_{k} \\
u_{k}
\end{array}\right) .
$$

Once we count all possible values of $u_{j}$ in the $j$ th slot, then the $v_{j}$ 's will fall into place, since the middle value in the tri-partition is $i_{j}$ and the total is $n_{j}$. Then

Lemma 5.2. The total number of terms in the substitution

$$
\left\{M_{(n)}\right\}\left\{N_{\left(i_{1}|\cdots| i_{k}\right)}\right\}\left\{a_{1}^{(1)} \cdots a_{n_{1}}^{(1)}|\cdots| a_{1}^{(k)} \cdots a_{n_{k}}^{(k)}\right\},
$$

where $n=\sum n_{j}-\sum i_{j}+1=\sum u_{j}+\sum v_{j}+1$, is

$$
\begin{aligned}
& \sum_{u_{1}=0}^{n_{1}-i_{1}} \cdots \sum_{u_{k}=0}^{n_{k}-i_{k}} \\
& \left(\begin{array}{c}
u_{1}+\cdots+u_{k} \\
u_{1}
\end{array}\right)\left(\begin{array}{c}
u_{2}+\cdots+u_{k} \\
u_{2}
\end{array}\right) \cdots\left(\begin{array}{c}
u_{k-1}+u_{k} \\
u_{k-1}
\end{array}\right)\left(\begin{array}{c}
u_{k} \\
u_{k}
\end{array}\right) \\
& \cdot\left(\begin{array}{c}
v_{1}+\cdots+v_{k} \\
v_{1}
\end{array}\right)\left(\begin{array}{c}
v_{2}+\cdots+v_{k} \\
v_{2}
\end{array}\right) \cdots\left(\begin{array}{c}
v_{k-1}+v_{k} \\
v_{k-1}
\end{array}\right)\left(\begin{array}{c}
v_{k} \\
v_{k}
\end{array}\right)
\end{aligned}
$$

(we are given only the $i_{j}$ 's and $n_{j}$ 's; note that $v_{j}=n_{j}-u_{j}-i_{j}$ in the formula).

When the type $(n)$ of the leftmost map is replaced by a generic partition $\pi$, things get a bit more complicated. We will simply stop here and refer the reader to the source, [3], as a much more precise method for this procedure is described in Subsection 2.2.2. So far, the reason for finer partitioning among the arguments of a multilinear map is not apparent. When barred braces are used for substitution, though, the types of multilinear functions that are produced play a prominent role in writing homotopy algebra identities.

\section{Appendix B: Properties of VOA's and TVOA's}

A vertex operator algebra (VOA) is a $\mathbf{Z}$-weighted (possibly with additional super $\mathbf{Z}$-grading, denoted by superscripts; not assumed here) module

$$
V=\bigoplus_{n} V_{n}
$$

of the Virasoro algebra that satisfies the following properties:

P1. $V_{n}=0$ for $n<<0$.

P2. There exists a linear map

$$
V \rightarrow \operatorname{End}(V)\left[\left[z, z^{-1}\right]\right], \quad v \mapsto v(z)=\sum v_{n} z^{-n-1},
$$


where $z$ is a formal variable, $v$ is called a state, and $v(z)$ is called a vertex operator (or a field). The linear map $v_{n}$ is called a mode of the vertex operator $v(z)$. We will sometimes denote the product $v_{n} w$ by $v \times_{n} w$.

P3. For all $v, w \in V$, we have $v_{n} w=0$ for $n>>0$.

P4. There exists an element $\mathbf{1} \in V_{0}$ such that

$$
\mathbf{1}(z)=\mathrm{id} \cdot z^{0} .
$$

P5. There exists $\omega \in V_{2}$ such that

$$
\omega(z)=\sum L_{n} z^{-n-2},
$$

where $L_{n}$ denotes both an element of $\operatorname{Vir}$ and the corresponding mode in $\operatorname{End}(V)$.

P6. For all $v \in V$, we have

$$
v(z) \mathbf{1} \in V[[z]]
$$

and

$$
\lim _{z \rightarrow 0} v(z) \mathbf{1}=v .
$$

P7. The operator $L_{0}$ is diagonalizable, with

$$
L_{0} v=(w t v) v \text { for all } v \in V_{n}
$$

(the eigenvalues are called weights).

P8. We have

$$
\frac{d}{d z} v(z)=\left(L_{-1} v\right)(z) \text { for all } v \in V .
$$

P9. Bracketing with $L_{0}$ results in

$$
\left[L_{0}, v(z)\right]=\left(L_{0} v\right)(z)+z\left(L_{-1} v\right)(z) \text { for all } v \in V .
$$

P10. For all $v, w \in V$, there exists an integer $t>>0$ such that

$$
\left[v\left(z_{1}\right), w\left(z_{2}\right)\right]\left(z_{1}-z_{2}\right)^{t}=0 .
$$

Some additional properties that follow from the definition are:

$\star\left(u_{m} v\right)_{n}=\sum_{i \geqslant 0}(-1)^{i}\left(\begin{array}{c}m \\ i\end{array}\right)\left[u_{m-i} v_{n+i}-(-1)^{m} v_{m+n-i} u_{i}\right]$

$\star\left[u_{m}, v_{n}\right]=\sum_{i \geqslant 0}(-1)^{i}\left(u_{i} v\right)_{m+n-i}$

$\star\left(\begin{array}{c}0 \\ i\end{array}\right)= \begin{cases}1 & \text { if } i=0 \\ 0 & \text { if } i \geqslant 1\end{cases}$

$\star\left(\begin{array}{c}-1 \\ i\end{array}\right)=(-1)^{i} \quad(i \geqslant 0)$

$\star\left(\begin{array}{c}m \\ i\end{array}\right)=\left\{\begin{array}{l}1 \text { if } i=0 \\ m(m-1)(m-2) \cdots(m-i+1) / i ! \quad \text { if } i \geqslant 1\end{array}\right.$

for $m \in \mathbf{Z}$

$\star L_{0}-(n+1) \cdot$ id is a derivation of $\times_{n}\left(L_{0}\right.$ is a derivation of Wick $)$

$\star\left[L_{0}, u_{n}\right]=(w t u-n-1) \cdot u_{n}$

$\star\left(u_{0} v\right)(z)=\left[u_{0}, v(z)\right]=\sum\left[u_{0}, v_{n}\right] z^{-n-1}$ 
$\star \frac{d}{d z} \sum u_{n} z^{-n-1}=\left(L_{-1} u\right)(z)=\sum\left[L_{-1}, u_{n}\right] z^{-n-1}=-\sum n u_{n-1} z^{-n-1}$

$\left(L_{-1}\right.$ is also a derivation of Wick)

$\star$ The mode $u_{n}$ is a differential operator of order $\leqslant n+1$ with respect to Wick for $n \geqslant 0$

$\star$ Wick is left pre-Lie, or $u_{-1} v_{-1}-v_{-1} u_{-1}-\left(u_{-1} v\right)_{-1}+\left(v_{-1} u\right)_{-1}=0$

$\star\left(u_{m} \mathbf{1}\right)_{n}$ has at most two terms:

$(-1)^{-n-1}\left(\begin{array}{c}m \\ -n-1\end{array}\right) u_{m+n+1}$ if $n \leqslant-1$ and $(-1)^{n}\left(\begin{array}{c}m \\ m+n+1\end{array}\right) u_{m+n+1}$ if $m+$ $n \geqslant-1$

$\star$ For a VOSA with nonnegative weights, $V_{0}$ is a (super) commutative, associative algebra under Wick

$\star$ For $w t u=m$ and $w t v=n$, all expressions $u_{t} v$ are zero for $t \geqslant m+n$-minimal weight

$\star$ A residue (charge) $u_{0}$ is a derivation of all binary products $\times_{n}$ :

$\left[u_{0}, \times_{n}\right](v, w)=u_{0}\left(v_{n} w\right) \pm\left(u_{0} v\right)_{n} w \pm v_{n}\left(u_{0} w\right)=0$

$\star$ Again if $u_{0}$ is a residue, we have

$u_{0} v_{n} \mathbf{1}=\left[u_{0}, v_{n}\right] \mathbf{1}=\left(u_{0} v\right)_{n} \mathbf{1}$ and $u_{0} v_{m} w_{n} \mathbf{1}=\left(u_{0} v\right)_{m} w_{n} \mathbf{1} \pm v_{m}\left(u_{0} w\right)_{n} \mathbf{1}$

$\star u_{n}=\frac{1}{n+1}\left[L_{-1}, u_{n+1}\right]$ for $n \neq-1$.

A topological vertex operator algebra (TVOA) is a super graded VOA with the following additional properties:

P11. There exists an even vertex operator $F(z) \in V_{1}$ whose residue $F_{0}$ is the fermion (ghost) number operator, or the super degree operator

P12. There exists a weight-one primary (Virasoro-singular) vertex operator $J(z)$ with fermion number one and a square-zero residue $Q=J_{0}$

P13. There exists a weight-two primary operator $b(z)=\sum b_{n} z^{-n-2}$ with fermion number -1 , satisfying

$$
[Q, b(z)]=\omega(z)
$$

\section{References}

[1] F. Akman, On some generalizations of Batalin-Vilkovisky algebras, J. Pure Appl. Algebra 120 (1997), 105-141.

[2] F. Akman, Multibraces on the Hochschild space, J. Pure Appl. Algebra 167 (2002), 129-163.

[3] F. Akman, A master identity for homotopy Gerstenhaber algebras, Commun. Math. Phys. 209 (2000), 51-76.

[4] C. Dong and J. Lepowsky, Generalized Vertex Algebras and Relative Vertex Operators, Progress in Mathematics 112, Boston, Birkhäuser, 1993.

[5] C. Dong, H. Li, and G. Mason, Vertex Lie algebra, vertex Poisson algebra and vertex algebras, Contemp. Math. 297 (2002), 69-96.

[6] M. Gerstenhaber, The cohomology structure of an associative ring, Ann. Math. 78 (1963), 267-288. 
[7] M. Gerstenhaber and A. A. Voronov, Higher order operations on Hochschild complex, Functional Anal. Appl. 29 (1) (1995), 1-6.

[8] E. Getzler, Cartan homotopy formulas and the Gauss-Manin connection in cyclic homology, Israel Mathematical Conference Proceedings 7 (1993), 65-78.

[9] E. Getzler and J. D. S. Jones, $A_{\infty}$-algebras and the cyclic bar complex, Ill. Jour. Math. 34 (1989), 256-283.

[10] Y.-Z. Huang and W. Zhao, Semi-infinite forms and topological vertex operator algebras, Comm. Contemp. Math. 2 (2000), 191-241.

[11] J. D. S. Jones, Lectures on operads. In Quantization, Poisson Brackets and Beyond (T. Voronov ed.), Contemporary Mathematics 315 (2002), 89-130.

[12] T. V. Kadeishvili, O kategorii differentialnych koalgebr i kategorii $A(\infty)$ algebr, Trudy Tbiliss. Mat. Inst. Razmadze Akad. Nauk Gruzin. SSR $\mathbf{7 7}$ (1985), 50-70, in Russian.

[13] T. Kimura, A. A. Voronov, and G. J. Zuckerman, Homotopy Gerstenhaber algebras and topological field theory. In: J.-L. Loday, J. Stasheff, and A. A. Voronov (eds.), Operads: Proceedings of Renaissance Conferences, Contemp. Math. 202 (1996), 305-333.

[14] J. Kock, Frobenius Algebras and 2D Topological Quantum Field Theories, London Mathematical Society Student Texts 59, Cambridge University Press, Cambridge, 2003.

[15] J.-L. Koszul, Crochet de Schouten-Nijenhuis et cohomologie, Astérisque (1985), 257-271.

[16] O. Kravchenko, Deformations of Batalin-Vilkovisky algebras, Banach Center Publications, Institute of Mathematics, Polish Academy of Sciences, Warszawa, 1999.

[17] T. Lada and M. Markl, Strongly homotopy Lie algebras, Comm. Algebra 23(6) (1995), 2147-2161.

[18] T. Lada and J. Stasheff, Introduction to SH Lie algebras for physicists, Internat. J. Theoret. Phys. 32(7) (1993), 1087-1103.

[19] B. H. Lian and G. J. Zuckerman, New perspectives on the BRST-algebraic structure of string theory, Commun. Math. Phys. 154 (1993), 613-646.

[20] M. Markl, A cohomology theory for $A(m)$-algebras and applications, J. Pure Appl. Algebra 83 (1992), 141-175.

[21] M. Markl, Homotopy algebras are homotopy algebras, Forum Mathematicum 16 (2004), 129-160.

[22] M. Penkava and A. Schwarz, On some algebraic structures arising in string theory. In: Perspectives in Mathematical Physics, Conf. Proc., Lecture Notes in Math. Phys. III, Cambridge, MA, International Press, 1994.

[23] M. Schlessinger and J. Stasheff, The Lie algebra structure of tangent cohomology and deformation theory, J. Pure Appl. Algebra 38 (1985), 313-322.

[24] J. D. Stasheff, Homotopy associativity of H-spaces, I, Trans. Amer. Math. Soc. 108 (1963), 275-292. 
[25] J. D. Stasheff, Homotopy associativity of H-spaces, II, Trans. Amer. Math. Soc. 108 (1963), 293-312.

[26] D. Tamarkin and B. Tsygan, Noncommutative differential calculus, homotopy BV algebras, and formality conjectures, Methods of Functional Analysis and Topology 6(2) (2000), 85-100.

[27] E. B. Vinberg, A Course in Algebra, Graduate Studies in Mathematics 56, American Mathematical Society, Providence, RI, 2003.

[28] A. A. Voronov, Homotopy Gerstenhaber algebras, Conference Moshe Flato 1999 (G. Dito and D. Sternheimer, eds.), vol. 2, Kluwer Academic Publishers, the Netherlands, 2000, 307-331.

This article may be accessed via WWW at http://www.rmi.acnet.ge/hha/ or by anonymous ftp at

$$
\text { ftp://ftp.rmi.acnet.ge/pub/hha/volumes/2005/n2a1/v7n2a1.(dvi,ps,pdf) }
$$

Füsun Akman akmanf@ilstu.edu

Department of Mathematics

313 Stevenson Hall

Campus Box 4520

Illinois State University

Normal, IL 61790 